\title{
Index numbers and decomposition spaces
}

\author{
Heikki Pursiainen \\ University of Helsinki and HECER
}

Discussion Paper No. 245

November 2008

ISSN 1795-0562

HECER - Helsinki Center of Economic Research, P.O. Box 17 (Arkadiankatu 7), FI-00014 University of Helsinki, FINLAND, Tel +358-9-191-28780, Fax +358-9-191-28781, E-mail info-hecer@helsinki.fi, Internet www.hecer.fi 


\title{
Index numbers and decomposition spaces*
}

\begin{abstract}
A price index is often loosely defined as some sort of average or mean of price ratios. A basic property of a mean, such as the arithmetic or geometric, for example, is that a grand mean may always be calculated as a suitably weighted mean of submeans. An analogous property of index numbers is called consistency in aggregation in the relevant literature. In this article we use the fact that with weak regularity conditions consistency in aggregation implies that price-quantity index pairs may be viewed as quasilinear means. Using this we show that these index number pairs may be equated with convex combinations in a suitably constructed vector space. We may thus apply linear algebra to their study, which is a completely new approach to the subject.

To illustrate the strength of our approach, we derive a equivalence theorem of quasilinear index number formulas and additive decompositions of value change. We argue that this result shows that consistency in aggregation may be interpreted as consistency between the treatment of ratios and di ${ }^{*}$ erences. We also show that many well-known index number axioms have linear algebraic interpretations and give conditions under which a decomposition space has a natural inner product structure.
\end{abstract}

JEL Classification: C43.

Keywords: Aggregation, index number theory, algebra, linear algebra.

Heikki Pursiainen

Department of Economics

University of Helsinki

P.O. Box 17

FI-00014 University of Helsinki

FINLAND

e-mail: heikki.pursiainen@helsinki.fi

*This article contains material from my $2005 \mathrm{PhD}$ dissertation titled "Consistent Aggregation Methods and Index Number Theory". 


\section{Introduction}

A price index is often loosely defined as some sort of average or mean of price ratios. A basic property of a mean, such as the arithmetic or geometric, for example, is that a grand mean may always be calculated as a suitably weighted mean of submeans. An analogous property, usually called consistency in aggregation by index number theorists (see e.g. Vartia (1976) Diewert (1978), Blackorby and Primont (1980), and Balk (1996)), may be defined for index number formulas. In this article we show, following Pursiainen (2007) that coupled with some basic regularity conditions consistency in aggregation implies that there is an exact sense in which price-quantity index pairs may be viewed as two-dimensional means. To be more precise, we give sufficient conditions for an index number pair to have a quasilinear mean representation (see Aczél (1966) as well as Pursiainen (2007) for discussion of quasilinear means).

We then proceed to show that quasilinear number pairs are in fact base-period expenditure -weighted convex combinations in a suitably chosen two-dimensional vector space. We give a characterization of such spaces, which we call decomposition spaces.

Equating quasilinear index number pairs with convex combinations makes it possible to apply linear algebra to their study. Doing this, we derive a fundamental equivalence theorem of quasilinear index number pairs and additive decompositions of value change. A two-dimensional quasilinear mean is an index number pair if and only if it may be derived from an additive decomposition. We argue that this result shows that quasilinearity (or consistency in aggregation) can be interpreted as consistency between the treatment of ratios and differences. This is an important property which is not shared by more complex index number formulas.

We then give conditions under which we may link each index number pair with a unique additive decomposition. The necessary and sufficient condition is that both indices satisfy the so-called identity test, requiring that no change in prices or quantities implies unit value for the corresponding index. We also show that the conditions imply an inner product, i.e. length and angle, for the corresponding decomposition space. The inner product structure is used among 
other things to represent the spaces associated with certain well-known formulas graphically.

In addition, we derive the implications for quasilinear indices of the so-called reversal axioms. The factor reversal test is shown to imply that the additive decomposition associated with it is symmetric with respect to prices and quantities. Similarly, the factor reversal is equivalent to a basic symmetry of the corresponding decomposition space. The time reversal test also implies analogous symmetries for the corresponding additive decomposition as well as the corresponding decomposition space.

We also illuminate the relation of index-number-theoretic axioms and linear algebra by including a section characterizing two well-known quasilinear indices, namely the Stuvel (1957) and Montgomery-Vartia (see Vartia (1976)) formulas.

The article is organized as follows. In Sections $2-3$ are preliminary sections describing the basic linear algebraic apparatus and theory of means necessary for the rest of the article. In Section 4 we show how index number pairs may be represented as means and in the following Section 5 the fundamental isomorphism of quasilinear index number formulas and additive decompositions is derived. Section 6 deals with the uniqueness of the additive decomposition and identity test, Section 7, describe the implications of the time and factor reversal tests. In Section 8 we discuss the structure of the Stuvel and Montgomery-Vartia formulas. There is also a brief final section giving some concluding remarks.

The results we present as formal theorems and corollaries in Sections 4-9. are mostly intuitive and concrete results about the connection of index numbers and additive decompositions and thus of interest to any reader familiar with basic index number theory. However, there is much material in the discussion about the linear algebra of index numbers which we find as important and interesting as the rest, but this may well not apply for the general reader. Similarly, as mentioned, the development of the linear algebraic apparatus has necessitated the inclusion of a preliminary technical part, contained in Sections 2 and 3. These sections contain definitions and results essential for the proof of our results, but may be too detailed and technical for all readers.

Because of these considerations, the article may be read in essentially two ways. The reader not interested in detailed linear algebraic discussion of the 
structure of index numbers may simply skip Sections $2-3$ and just read through the theorems and the discussion immediately around them. This should give the reader an idea about the more concrete implications of our results. However, a reader more interested in the structure of means and index number formulas, should definitely read the introductory sections as well as the proofs in the appendix because these contain material that is of interest in itself. Also, while the formal theorems are mostly given in the language of index numbers and additive decompositions, all sections contain material on the linear algebraic structure of decomposition spaces that should interest theoretically minded readers.

\section{Quasilinear and arithmetic means}

The purpose of this section is to establish an equivalence between quasilinear means and convex combinations in finite-dimensional linear spaces. By a linear space we always mean a linear space over the real numbers. The weighted arithmetic mean or simply the weighted mean in a linear space $L$ is defined in the usual way. For any strictly positive weights $\lambda_{i}$ and vectors $x_{i} \in L$ the arithmetic mean $\bar{x}$ is the convex combination $\bar{x}=\alpha_{1} x_{1}+\ldots+\alpha_{n} x_{n}$ with the coefficients $\alpha_{i}=\lambda_{i} / \sum_{j=1}^{n} \lambda_{j}$. For the standard arithmetic mean we may take $L=\mathbb{R}$ interpreted as the one-dimensional Euclidean space. However, the definition applies equally well to any linear space $L$.

A related class of means often encountered in applications is the class of quasilinear means. We use the following rather broad definition for a quasilinear mean. Let $A$ be a set and let $f: A \rightarrow C$ be a bijection from $A$ to some convex $k$-dimensional subset $C$ of $\mathbb{R}^{k}$. Then the weighted quasilinear mean of elements $x_{i} \in A$ defined by $f$, is given by $\bar{x}_{f}=f^{-1}\left[\sum_{i=1}^{n} \alpha_{i} f\left(x_{i}\right)\right]$, with coefficients $\alpha_{i}$ as above. The standard case would be $A=\Omega$ where $\Omega$ denotes the set of strictly positive reals with $C$ some real interval. However, this definition also covers a much broader class of means. We call the function $f$ a representation function or in an abbreviated form simply a representation of the corresponding mean. This is because, as is well-known, the same mean may be represented by different functions. The dimensionality of a quasilinear mean is the dimension of $C$. 
A simple one-dimensional example is provided by the geometric mean. Choose $A=\Omega$ and $f=\log$. This results in the geometric mean or $\bar{x}_{\log }=x_{1}^{\alpha_{1}} \ldots$ $x_{n}^{\alpha_{n}}$. The geometric mean is an exponentially-weighted product of the $x_{i}$ 's. It is trivial to see that we may make the set $\Omega$ a one-dimensional linear space by interpreting exponentiation as scalar multiplication and multiplication as vector addition. Clearly the geometric mean coincides with the arithmetic mean (i.e. convex combination) of this multiplicative space. It is also trivial to see that the natural logarithm function may be interpreted as a linear bijection from the space on $\Omega$ to the one-dimensional Euclidean space $\mathbb{R}$. This raises the question whether a similar result holds in general. That is, whether for any quasilinear mean there in fact exists some linear space $L$ such that the quasilinear mean in question coincides with convex combination in this space. The answer is yes, and establishing this result and its corollaries is the purpose of this section.

We call a finite-dimensional linear space $L$ an extension of the set $A$ if there exists a convex full-dimensional subset $A^{\prime}$ of $L$ and a bijection $f: A \rightarrow A^{\prime}$ and we have decided to equate the two sets $A$ and $A^{\prime}$ by equating each $x \in A$ with its image $x^{\prime}=f(x)$. As the two sets are considered equal, we may as well say that $L$ is an extension of $A$ whenever $A$ is a convex full-dimensional subset of $L$.

Theorem 1 Any quasilinear mean on a set A coincides with the arithmetic mean of a suitably chosen extension $L$ of $A$.

Conversely, the arithmetic mean of any extension $L$ of $A$ coincides with a quasilinear mean on A.

Two extensions $L$ and $L^{\prime}$ of $A$ are considered affine transformations of each other if there exists an affine map $J: L \rightarrow L^{\prime}$ such that the restriction $\left.J\right|_{A}$ coincides with the identity map of $A$.

As a special case of this, two extensions $L$ and $L^{\prime}$ of $A$ are considered equal if there exists a bijective linear map $I: L \rightarrow L^{\prime}$ such that the restriction $\left.I\right|_{A}$ is the identity map of $A$. Then we equate $I$ with the identity map of $L$ and write $L=L^{\prime}$.

Let $g: A \rightarrow C, \quad f: A \rightarrow C^{\prime}$ be bijections from $A$ to $k$-dimensional convex sets $C, C^{\prime} \subset \mathbb{R}^{k}$ respectively. Then we say that $g$ is an affine transformation of $f$ if there exists a non-singular $k \times k$ matrix $B$ and a vector $y_{0} \in \mathbb{R}^{k}$ such that 
$g(x)=B\left[f(x)-y_{0}\right]$ for all $x$. As a special case, $g$ is a linear transformation of $f$ if $y_{0}=0$.

With these definitions we may move on to the next theorem, which lists certain properties of extensions that we need to proceed.

Theorem 2 Let $L$ be an extension of a set $A$ such that the mean of $L$ coincides with the quasilinear mean represented by $f: A \rightarrow C \subset \mathbb{R}^{k}$.

1. $\operatorname{dim}(L)=k$.

2. The function $g: A \rightarrow \mathbb{R}^{k}$ represents the same quasilinear mean as $f$ iff it is an affine transformation of $f$.

3. The extension $L$ is unique up to an affine transformation.

4. L may always be chosen so that there exists a linear bijection $\hat{f}: L \rightarrow \mathbb{R}^{k}$ such that $f=\left.\hat{f}\right|_{A}$. This $L$ is unique given $f$ and is called the $f$-extension.

5. If $L$ is the $f$-extension, then $\hat{f}$ is a coordinate function corresponding to a basis of L. This implies:

(a) If the origin $0 \in L$ of the $f$-extension is in the subset $A \subset L$, then it is given by the equation $f(0)=0$.

(b) Let $u_{1}, . ., u_{k} \in L$ be the basis vectors corresponding to the coordinate function $\hat{f}$. If $u_{i}$ is in the subset $A \subset L$ for some $i$ then $f\left(u_{i}\right)=e_{j}$, where $e_{j} \in \mathbb{R}^{k}$ is a canonical basis vector.

(c) If $g$ is an affine transformation of $f$ then the $g$-extension is an affine transformation of the $f$-extension.

(d) If $g$ is a linear transformation of $f$ then the $g$-extension is same as the $f$-extension and $f$ and $g$ are simply restrictions of coordinate functions corresponding to two different bases of the $f$-extension. 


\section{An example: the Pythagorean means}

The well-known Pythagorean means, i.e. the arithmetic, the geometric and the harmonic mean defined on the positive reals $\Omega$, provide a nice and simple example of the rather abstract results presented in the previous section.

All three are one-dimensional quasilinear means. The arithmetic mean may be represented by the function $f: \Omega \rightarrow \Omega, f(x)=x$, the geometric mean by the natural logarithm as already mentioned, while the harmonic mean may be represented by $h(x)=\frac{1}{x}$, with $h$ mapping $\Omega$ to $\Omega$. All map the set $\Omega$ to a real interval and thus are one-dimensional. By Theorem 1 each of the three means is the mean of some linear space extension of $\Omega$. Point 1 in Theorem 2 implies that the dimension of each extension is 1 .

Consider first the arithmetic mean. The $f$-extension space of point 4 in Theorem 2 for $f(x)=x$ is $\mathbb{R}$ as the standard one-dimensional Euclidean space, as clearly $\hat{f}(x)=x$ with the domain extended to all $\mathbb{R}$ is the required coordinate function extension of $f$. The class of affine transformations of $f$ is simply the class of non-constant affine maps $f_{a, b}(x)=a+b x, b \neq 0$ defined for $x>0$. It is clear that any of these is also a representation of the arithmetic mean as Theorem 2 requires. Also, for any $f_{a, b}$ the linear extension $\hat{f}_{a, b}$ is simply the same affine map with the domain of definition extended to cover all real numbers. The corresponding $f_{a, b}$-extensions are affine transformations of the standard $\mathbb{R}$, i.e. simply $\mathbb{R}$ but with the origin possibly shifted away from 0 . Point 5 of Theorem 2 in this case simply verifies obvious facts about the standard $\mathbb{R}$, for example that the origin of $\mathbb{R}$ is 0 , that $\hat{f}(x)=x$ is the coordinate function for basis $u=1$ etc.

The geometric mean was already discussed in the beginning of the previous section. Putting this discussion in terms of Theorems 1 and 2 it directly implies that the log-extension of $\Omega$ is $\Omega$ itself with exponentiation as scalar multiplication and multiplication as vector addition. This of course implies also that $\log =\widehat{\log }$. Also, $\log$ is the coordinate function for basis $e \in \Omega$ of $\Omega$. Similarly, the origin of $\Omega$ is 1 .Any affine transformation of the logarithm function, i.e., any function of the form $g_{a, b}(x)=a+b \log x$ with constants $a$ and $b \neq 0$ also represents the geometric mean, with any $g_{a, b}$ being the coordinate function of an affine transformation of $\Omega$. 
The harmonic mean may be represented by $h(x)=\frac{1}{x}$. The $h$-extension of $\Omega$ is a one-dimensional linear space $L$ containing $\Omega$ as a convex subset. But in contrast to the arithmetic and geometric means, there is no natural way of identifying $L$ with $\mathbb{R}$ or some other well-known set. It is simply a space defined on a set consisting of certain equivalence classes some of which are identified with elements in $\Omega$ and some are not. There is no intuitive way of saying what vectors in $L$ outside the set $\Omega$ "look like". Also, $h$ is not equal to zero for any $x>0$. Thus the origin of $L$ lies outside of $\Omega$. As $h(1)=1$, the relevant basis of the $h$-extension space is 1 .

All three extension spaces share the common subset $\Omega$. Indeed the extension space of the geometric mean is completely contained in in the extension space corresponding to the arithmetic mean. In contrast the vectors lying outside $\Omega$ in the extension space of the harmonic mean cannot be equated in any simple way with real numbers. In a sense, we are not really interested on the vectors outside $\Omega$ but we just need to know that they are there to be able to treat the harmonic mean as a convex combination. This is true about our treatment of index numbers as well. We will give conditions under which index numbers formulas may be treated as convex combinations in a two-dimensional space. In this case the spaces will be extensions of $\Omega^{2}$. Again, we will not be really interested in the vectors outside this set but they are necessary for the linear algebraic treatment.

Another point that is illuminated by the Pythagorean means is the nonuniqueness of representation. Any affine transformation of the functions $f, \log$ or $h$ may be used to represent the same mean as the original ones. In this sense they are completely equivalent and there are no reason or criteria for discriminating between them. However, from the linear algebraic point of view the choice of representation implies a choice of origin and basis for the extension space. Thus there may sometimes exist reasons for preferring some representation over another. If it is possible to give an intuitive interpretation for one representation which the others lack, it is reasonable to adopt this representation as a "canonical" one. For example if we are dealing with aggregating ratios of two variables using Pythagorean means, such a criterion might be that we would like the representations to be "normed indicators of relative change" in the sense of Törnqvist, Vartia and Vartia (1985). In this case we would be led to choose the 
representations $f_{-1,1}(x)=x-1, \log$ and $h_{1,-1}(x)=1-\frac{1}{x}$ for the arithmetic, geometric and harmonic means respectively. These choices imply a choice of basis and origin for each extension space which we could then adopt as natural for the study of ratios or relative change.

\section{Index numbers as two-dimensional means}

The well-known index number problem concerns aggregation of price and quantity data collected on a finite number of commodities on two time periods, $t=0,1$. We use the notation $p_{i}^{t}$ and $q_{i}^{t}$ for the price and quantity of the $i$ th commodity on period $t$. We define a price index formula to be a rule mapping any finite collection of prices and quantities for the two periods to a price index $P$, which is a positive real number. Following convention ${ }^{1}$ we postulate that the price index formula depends on the measurements only via the price ratios $^{2} x_{i}=\frac{p_{i}^{1}}{p_{i}^{0}}$ and the values or expenditures $v_{i}^{t}=p_{i}^{t} q_{i}^{t}$. That is, a price index formula may be represented via a sequence of functions $g_{n}$ where $n$ corresponds to the number of commodities so that for any collection of price-quantity data for $n$ commodities the price index is given by $P=g_{n}\left(\left(x_{1}, v_{1}^{0}, v_{1}^{1}\right), \ldots,\left(x_{n}, v_{n}^{0}, v_{n}^{1}\right)\right)$.

A price index formula defines implicitly a quantity index formula via the requirement that the product of the price and quantity indices should yield the aggregate value or expenditure ratio, i.e. that $P Q=\frac{V^{1}}{V^{0}}$ always. Here we have used the notation $V^{t}=\sum_{i} v_{i}^{t}$ for the aggregate expenditure on period $t$. It should be clear that a quantity index formula thus derived may be represented as a sequence of functions similarly to the price index formula. Together, a price index formula and the quantity index formula derived from it are called an index number pair.

To be able to apply the theory of quasilinear means to index numbers we have to diverge somewhat from the above, standard representation. First, note

\footnotetext{
${ }^{1}$ I.e. we assume that the price index satisfy the so-called commensurability axiom, see e.g. Balk (1996) for discussion.

${ }^{2}$ The symbols $x_{i}$ and $y_{i}$ are used for the price and quantity relatives respectively instead of the more standard $\pi_{i}$ and $\kappa_{i}$, because we have reserved the Greek alphabet for scalars in the vector space notation. For the same reason, we denote the expenditure shares $\alpha_{i}^{0}=\frac{v_{i}^{0}}{V^{0}}$ instead of the more familiar $w_{i}^{0}$ or $s_{i}^{0}$.
} 
that the triple $\left(x_{i}, v_{i}^{0}, v_{i}^{1}\right)$ may always be bijectively mapped to $\left(x_{i}, y_{i}, v_{i}^{0}\right)$, where $y_{i}=\frac{q_{i}^{1}}{q_{i}^{0}}$ is the quantity ratio or relative. Thus, we can equally well represent the index number pair as a sequence of two-dimensional functions, so that

$$
(P, Q)=h_{n}\left(\left(x_{1}, y_{1}, v_{1}^{0}\right), \ldots,\left(x_{n}, y_{n}, v_{n}^{0}\right)\right)
$$

for any data from $n$ commodities. By construction, $(P, Q)$ satisfies

$$
P Q=\frac{V^{1}}{V^{0}}
$$

Conversely, it is clear that (1) defines an index number pair only if (2) is satisfied. Thus the two equations may actually be used as a alternative definition of an index number pair.

Note that one possibility for the functional form of the functions $h_{n}$ is

$$
(P, Q)=d^{-1}\left[\sum_{i=1}^{n} \frac{v_{i}^{0}}{V^{0}} d\left(x_{i}, y_{i}\right)\right]
$$

where $d$ maps $\Omega^{2}$ bijectively to a convex subset $C$ of the plane. In this case the index number pair is a two-dimensional $v_{i}^{0}$-weighted quasilinear mean of the pricequantity ratio pairs. Not all quasilinear means on $\Omega^{2}$ are index number pairs, as it is necessary for $(P, Q)$ to satisfy $(2)$. But clearly some index number pairs have the required form. For example, simple calculation shows that choosing $d(x, y)=$ $(x-1, x y-x)$ results in the well-known Laspeyres price index - Paasche quantity index pair (see Table 1 below for more examples).

Index number pairs that can be represented as (3) are said to be quasilinear. If the representation $d$ may be chosen to be continuous then the index number pair is said to have a continuous quasilinear representation.

In short, a quasilinear index number pair is a two-dimensional quasilinear mean on the set $\Omega^{2}$ which also satisfies the aggregate decomposition condition (2). These indices are the main object of study in this article. To show that the class of quasilinear formulas is in fact theoretically interesting we give a characterization based on a set of intuitively appealing axioms. This result is a special case of an extension given in Pursiainen (2007) of the classical characterization of one- 
dimensional quasilinear means of Nagumo (1930) and Kolmogorov (1930) (see also Aczél (1966)).

A basic property of the quasilinear mean form is consistency in aggregation. Loosely put this means consistency of the following two-step procedure. First, partition the commodities into subsets, then calculate the index number pair and the total base period expenditure in each of the subsets. In the next step, use the resulting subindices and expenditure subaggregates to calculate a grand mean or index number pair. It is an obvious feature of the quasilinear form that the result of the two-step procedure always agrees with the indices calculated directly from the price-quantity data. For a detailed description of the property see the Appendix and Pursiainen (2007).

Three other conditions along with consistency in aggregation are sufficient for a quasilinear form. The index number pair is called weakly proportional, if all price and quantity relatives are equal, so that $\left(x_{i}, y_{i}\right)=(x, y)$ for all $i$, then $(P, Q)=(x, y)$ regardless of the expenditures $v_{i}^{0}$. The index number pair is called one-to-one if, keeping the expenditures $v_{i}^{0}$ fixed, any price-quantity relative pair $\left(x_{j}, y_{j}\right)$ cannot be changed to some $\left(x_{j}^{\prime}, y_{j}^{\prime}\right) \neq\left(x_{j}, y_{j}\right)$ without the resulting index number pair $\left(P^{\prime}, Q^{\prime}\right)$ being unequal to the original value $(P, Q)$, so that $\left(P^{\prime}, Q^{\prime}\right) \neq(P, Q)$. The following theorem is a special case of Theorem 2 in Pursiainen (2007).

Theorem 3 An index number pair is quasilinear if it is (i) consistent in aggregation, (ii) continuous, (iii) one-to-one and (iv) weakly proportional. These sufficient conditions are also necessary for a continuous quasilinear representation to exist.

\section{Additive decompositions and index numbers}

Consider the set of positive reals $\Omega$ with the multiplicative structure defined above. The product of each price relative $x_{i}$ and a quantity relative $y_{i}$ is the expenditure relative $\frac{v_{i}^{1}}{v_{i}^{0}}=x_{i} y_{i}$. In other words, the expenditure ratio decomposes into a price and quantity ratio with respect to the multiplication operation in $\Omega$. Thus we may interpret all pairs $\left(x_{i}, y_{i}\right) \in \Omega^{2}$ as multiplicative decompositions or 
simply decompositions of expenditure ratios. The product set $\Omega^{2}$ is the set of all possible decompositions. Quasilinear index number pairs are quasilinear means defined on the set of decompositions with the additional property (2). We may interpret (2) as the requirement that an index number pair be a decomposition of the aggregate expenditure ratio whenever the individual price-quantity ratios are decompositions of the individual expenditure ratios. Thus, index number pairs are two-dimensional means characterized by this consistency with respect to multiplicative decomposition.

Because quasilinear index number pairs are quasilinear means we may use the linear algebraic apparatus developed in Section 2 to explore their properties. It follows from Theorem 1 and Point 1 of Theorem 2 that any quasilinear index number pair is the arithmetic mean of a suitably chosen two-dimensional space. It thus remains to characterize the decomposition property (2) in linear-algebraic terms. We will do this in two steps in this section. First, we give a technical description of the spaces, called decomposition spaces, which correspond to quasilinear index number pairs. In the second step, we use this result to prove an equivalence theorem between index number pairs and additive decompositions.

A decomposition space is a two-dimensional extension $D$ of the set of decompositions with the following properties: (i) it has origin $(1,1) \in \Omega^{2}$ called the null decomposition and (ii) there exists a linear functional $\hat{s}: D \rightarrow \mathbb{R}$, called the composition functional, such that the restriction ${ }^{3} s=\hat{s}_{\mid \Omega^{2}}$ satisfies $s(x, y)=x y-1$ for all $(x, y) \in \Omega^{2} \subset D$.

Lemma 1 A quasilinear mean on the set $\Omega^{2}$ is an index number pair iff it is coincides with the arithmetic mean of a decomposition space.

Decomposition spaces are two-dimensional linear spaces. Such spaces are always isomorphic to Euclidean spaces with the same dimension. This means that the basic properties of a decomposition space must have their exact counterparts in the isomorphic Euclidean spaces. For example, as the composition functional is a linear functional on $D$, then for any basis $U$ of $D$, there must be a corresponding

\footnotetext{
${ }^{3}$ Another way of putting this requirement would be that the multiplication operation in $\Omega^{2}$ must be a restriction of an affine map $D \rightarrow \mathbb{R}$. However, while this formulation would perhaps reflect the algebraic nature of the index number problem somewhat better it is nicer to work with linear functionals than affine ones.
} 
linear functional on $\mathbb{R}^{2}$ operating on the $U$-coordinates. This provides us with the means to prove what is perhaps our main result, the equivalence of additive decompositions of expenditure change and quasilinear index numbers.

Consider the problem of decomposing the absolute (as opposed to relative) expenditure change $v^{1}-v^{0}=\Delta v$ into a price and quantity component $\tilde{p}$ and $\tilde{q}$ so that $v^{1}-v^{0}=\tilde{p}+\tilde{q}$, where $\tilde{p}$ and $\tilde{q}$ are interpreted as additive measures of the contribution of prices and quantities respectively to the expenditure change. More precisely, let $m$ be a function of the price and quantity relatives and period 0 expenditure, consisting of two components $m_{1}$ and $m_{2}$ which identically satisfy $\Delta v=m_{1}\left(x, y, v^{0}\right)+m_{2}\left(x, y, v^{0}\right)$. The first component is interpreted as the price measure so that $\tilde{p}=m_{1}\left(x, y, v^{0}\right)$ the second one measures the quantity contribution so that $\tilde{q}=m_{2}\left(x, y, v^{0}\right)$. Of course, for these interpretations to be meaningful the function $m$ must satisfy additional criteria which we will discuss below. However, an immediate condition we impose is that $m$ should be linearly homogeneous with respect to $v^{0}$, as inflating all prices by the same factor should not result in a change in the decomposition. This condition implies that both $m_{i}$ must be of the form $m_{i}\left(x, y, v^{0}\right)=v^{0} d_{i}(x, y)$ for some functions $d_{i}$. Dividing the decomposition equation $v^{1}-v^{0}=v^{0} d_{1}(x, y)+v^{0} d_{2}(x, y)$ by $v^{0}$ it is seen to be equivalent to

$$
x y-1=d_{1}(x, y)+d_{2}(x, y) .
$$

Functions $d=\left(d_{1}, d_{2}\right)$ which have this property are called additive decomposition functions. By definition an additive decomposition function is any function which maps all multiplicative decompositions to a corresponding additive decomposition. One of our main results is the following connection between additive decomposition functions and quasilinear indices:

Theorem 4 A quasilinear mean on $\Omega^{2}$ is an index number pair iff it has a representation $d$ which is an additive decomposition of expenditure change.

In other words, all additive decompositions define a quasilinear index number formula and vice versa. Put more forcefully, additive decompositions and quasilinear index numbers are just different representations of the same thing. In the following, we will call an additive decomposition function which defines an index 


\begin{tabular}{|l|l|l|}
\hline Formula & $d_{1}(x, y)$ & $d_{2}(x, y)$ \\
\hline Laspeyres & $x-1$ & $x(y-1)$ \\
Paasche & $y(x-1)$ & $y-1$ \\
Geometric (or log-)Laspeyres & $\log x$ & $x y-1-\log x$ \\
Geometric (or log-)Paasche & $x y-1-\log y$ & $\log y$ \\
Stuvel (1957) & $\frac{1}{2}(x-1)+\frac{1}{2} y(x-1)$ & $\frac{1}{2}(y-1)+\frac{1}{2} x(y-1)$ \\
Montgomery-Vartia & $l(x y) \log x$ & $l(x y) \log y$ \\
\hline
\end{tabular}

Table 1: Additive decompositions corresponding to well-known formulas

number pair an additive decomposition representation or simply an additive representation for the index number pair. By the previous theorem, all quasilinear index number pairs have an additive decomposition representation. In Table 1, these representations are presented for certain well-known formula pairs. The list is not meant to be exhaustive. Each formula pair is named for the price index, as the functional form of the price and quantity index may differ. It is a matter of straightforward calculation to verify that each function in the list is indeed an additive decomposition and that substituting any of the functions into equation (3) does yield the index number pair for which it is named.

The linear algebraic interpretation of Theorem 4 is that any decomposition space has a basis the coordinate function of which is an additive decomposition function. This interpretation follows directly from Property 3 of Theorem 2.

On a more concrete level, Theorem 4 may be interpreted as giving a method of constructing quasilinear index numbers from additive decompositions and vice versa. For example, assume that it has been decided how expenditure changes should be decomposed additively into price and quantity components $\left(\tilde{p}_{i}, \tilde{q}_{i}\right)=$ $v_{i}^{0} d\left(x_{i}, y_{i}\right)$ with $v_{i}^{1}-v_{i}^{0}=\tilde{p}_{i}+\tilde{q}_{i}$. Such decompositions can be aggregated by simply adding them up into an aggregate decomposition, i.e. $V^{1}-V^{0}=\tilde{P}+\tilde{Q}$ where $(\tilde{P}, \tilde{Q})=\sum_{i=1}^{n} v_{i}^{0} d\left(x_{i}, y_{i}\right)$. Now, it is possible to define a price-quantity index number pair $(P, Q)$ by postulating a natural consistency requirement between the aggregate and commodity-level decompositions. As we have $\tilde{p}_{i}=v_{i}^{0} d_{1}\left(x_{i}, y_{i}\right)$ and $\tilde{q}_{i}=v_{i}^{0} d_{2}\left(x_{i}, y_{i}\right)$ for each individual commodity, the analogous identities $\tilde{P}=V^{0} d_{1}(P, Q)$ and $\tilde{Q}=V^{0} d_{2}(P, Q)$ should hold on the aggregate level. In other words, the values for the price index $P$ and the quantity index $Q$ should be equal to the price relative and quantity relative which would lead to the same 
aggregate additive decomposition as the adding up of the individual additive decompositions. But it is seen immediately that this consistency condition is simply the definition of a quasilinear mean represented by $d$. Theorem 4 guarantees that this mean is always an index number pair i.e. it satisfies (2).

\section{Uniqueness and the identity test}

A basic problem with Theorem 4 is that the additive representation of an index number pair is not unique. Suppose that we have chosen $d=\left(d_{1}, d_{2}\right)$ to satisfy $d_{1}(x, y)+d_{2}(x, y)=x y-1$. It is clear that a number of bijective linear transformations $\tilde{d}=C d$ of $d$ satisfy this linear restriction. By Theorem 2 any such transformation represents the same index number pair and simply corresponds to a change of basis of $D$.

To resolve the ambiguity of representation we need more structure. This is provided by the so-called identity test, which is a basic index-number-theoretic axiom. For a price index it is simply the requirement that if all prices have remained unchanged, the price index should equal unity. A similar axiom may be formulated, mutatis mutandis, for the quantity index. We say that the index number pair $(P, Q)$ satisfies the identity test if both indices satisfy the relevant identity test.

To see that the axiom thus formulated has a linear algebraic structure, define the subsets $E_{1}=\{(x, 1) \mid x \in \Omega\}$, and $E_{2}=\{(1, y) \mid y \in \Omega\}$ of a decomposition space $D$. Here $E_{1}$ is the subset of decompositions with no quantity change and $E_{2}$ similarly the subset of decompositions with no price change. For the index number pair to satisfy the identity test we must always have $\sum_{i=1}^{n} \alpha_{i}^{0}\left(x_{i}, 1\right)=(P, 1)$ and $\sum_{i=1}^{n} \alpha_{i}^{0}\left(1, y_{1}\right)=(1, Q)$ for some $P$ and $Q^{5}$. Clearly this is equivalent to requiring that the two sets $E_{1}$ and $E_{2}$ be convex in $D^{6}$. The identity test is thus a simple

\footnotetext{
${ }^{5}$ In fact, we must have $P=\frac{V^{1}}{V^{0}}$ in the first case and $Q=\frac{V^{1}}{V^{0}}$ in the second. This is because the index number pair must satisfy equation (2).

${ }^{6}$ Note that this is indeed an additional requirement, as it is not necessary for any particular properties of the set $\Omega^{2}$ to be transferred to the decomposition space $D$. In particular, while $\Omega^{2}$ is the Cartesian product of $\Omega$ with itself, generally $D$ has no "canonical" representation as a product of two sets or a direct sum of two subspaces. As the product structure is not preserved,
} 
convexity condition.

The identity test is directly related to an analogous condition of additive decomposition functions. As mentioned, we would like to interpret $d_{1}$ as an additive measure of the contribution of prices to expenditure change and $d_{2}$ as a similar measure for quantities. A minimal requirement for such an interpretation would seem to be that when the other factor contributes nothing to the product, i.e. when we have either $(x, y)=(x, 1)$ or $(x, y)=(1, y)$ the additive decomposition function should respect this. In other words, we should have $d_{2}(x, 1)=0$ and $d_{1}(1, y)=0$.That is, when the other side of the multiplicative decomposition contributes nothing to the product, the corresponding "side" of the additive decomposition should be normalized equal to zero. Of course this implies that in this case the whole expenditure change is attributed to the factor which has changed, so that for example $v^{0} d_{1}\left(\frac{v^{1}}{v^{0}}, 1\right)=v^{1}-v^{0}$. An additive decomposition function satisfying this basic property is said to be normalized.

Theorem 5 A quasilinear index number pair may be represented by a unique normalized additive decomposition function of expenditure change iff it satisfies the identity test.

The identity test is therefore all the additional structure that is needed to guarantee the existence of a unique representation. Given the connection between normalized additive decompositions, index number pairs which satisfy the identity test and the corresponding decomposition spaces it is natural to call all three normalized. As was seen in the proof, choosing the normalized representation $d$ corresponds to a choice of basis of the decomposition space $D$. It was shown that if the two sets $E_{1}$ and $E_{2}$ are convex they must in fact be one-dimensional convex sets, i.e. line segments in $D$. Moreover, the two segments are linearly independent and may be used to form a basis for $D$. Thus any normalized $D$ has an unique basis consisting of two vectors $x_{0}$ and $y_{0}$ which produces the normalized representation. The basis vector $x_{0}$ corresponds to a direction of pure price change and similarly $y_{0}$ to a direction of pure quantity change. As the first component $d_{1}$ in the additive representation is interpreted as a measure of the contribution of price change to the expenditure difference, it is reasonable to call this as giving subsets such as $E_{1}$ or $E_{2}$ need not be convex in $D$. 
the additive price coordinate of the vector $(x, y) \in \Omega^{2}$ and similarly call $d_{2}$ the additive quantity coordinate ${ }^{7}$.

In Table 1 listing examples of additive decompositions / quasilinear index number pairs we have given the unique normalized decomposition whenever it exists. It is straightforward to verify the well-known fact that among the formula pairs listed the Laspeyres-Paasche, Paasche-Laspeyres, the Stuvel and the Montgomery-Vartia pairs are normalized while index number pairs corresponding to the geometric Laspeyres and geometric Paasche price indices are not.

Note that the existence of a unique basis makes it possible to speak of length and angle in a normalized decomposition space. This may be done by endowing the space $D$ with the unique inner product structure in which the basis vectors corresponding to the normalized representation are orthonormal. In other words, we simply decide to interpret the linear extension $\hat{d}: D \rightarrow \mathbb{R}^{2}$ of the unique normalized decomposition as an inner product space isomorphism to $\mathbb{R}^{2}$ with the usual inner product. Thus, for normalized quasilinear indices it makes sense to speak of movements along a price axis and quantity axis, with the two axes orthogonal. Whenever we speak of length or angles in normalized decomposition spaces this is to be understood with respect to the normalized basis.

The inner product structure also induces the unique topology which makes $D$ a Hausdorff topological vector space. It is not too complicated an exercise to show that when the conditions of Theorem 3 are satisfied so that the additive decomposition function $d$ is a continuous function on $\Omega^{2}$ the linear space extension $D$ of $\Omega^{2}$ is topological in the sense that the topology that $D$ as an inner product space induces on $\Omega^{2} \subset D$ is the standard topology of $\Omega^{2}$ (see e.g. Pursiainen (2007)).

Still another implication of the inner product is that normalized spaces may be compared with respect to an equivalent orthogonal basis, i.e. the normalized basis. This implies that it is possible to represent the spaces graphically in a natural way on the plane by identifying the normalized basis vectors as the canonical basis vectors of $\mathbb{R}^{2}$ and then in turn identifying each decomposition $(x, y) \in \Omega^{2}$ with its coordinates in this basis. More concretely, letting $d$ be the normalized additive representation and $\hat{d}$ its linear extension we can identify each point $z \in D$ with $\hat{d}(z) \in \mathbb{R}^{2}$, in particular, each decomposition $(x, y) \in \Omega^{2} \subset D$ is identified

\footnotetext{
${ }^{7}$ The basis was shown in the proof to be given by $x_{0}=(2,1)$ and $y_{0}=(1,2)$.
} 
with its additive coordinates ${ }^{8} d(x, y) \in \mathbb{R}^{2}$.

We will use this graphical device to discuss the geometry of decomposition spaces with the aid of three normalized examples: the Laspeyres space corresponding to Laspeyres price index - Paasche quantity index number pair, the Stuvel space and the Montgomery-Vartia space corresponding to the decomposition functions in Table 1. All three index number pairs have different properties which fact is reflected by the different geometry of the corresponding decomposition spaces.

The graphical representations are given in Figures 1a., 1b. and 1c. ${ }^{9}$. In each case the shaded area represents the set of decompositions $\Omega^{2}$. Note that by construction this is always a full-dimensional convex subset of $D$. Also, the convex set $\Omega^{2}$ is always contained in the half-space $H=\{z \in D \mid \hat{s}(z)>-1\}$ defined by the requirement that the composition functional be greater than -1 . This follows from the definition of the composition functional. Recall that the restriction $s=\hat{s}_{\mid \Omega^{2}}$ is given by $s(x, y)=x y-1$. As the expenditure ratio $\frac{v^{1}}{v^{0}}=x y$ is positive, $\Omega^{2}$ must be contained in $H$. In the cases of the Stuvel and MontgomeryVartia formulas we have in fact $H=\Omega^{2}$. However, in the case of the Laspeyres price index, $\Omega^{2}$ is a strict, asymmetric subset of $H$. This reflects the basic lack of symmetry of the Laspeyres formula. In general, the level sets associated with the composition functional are important. In so far as they are contained in $H$ each level set is associated with all price and quantity ratios which imply a fixed expenditure ratio. Of particular interest is the kernel of $\hat{s}$, associated with no overall expenditure change. We will discuss this, as well as the additional features depicted in the figures, below.

\footnotetext{
${ }^{8}$ To put this in more abstract terms, we have two isomorphic inner product spaces, $D$ and $\mathbb{R}^{2}$, with the extension $\hat{d}$ of the additive representation $d$ providing the isomorphism. The graphical representation simply identifies each vector $z \in D$ with the corresponding vector $\hat{d}(z)$ in $\mathbb{R}^{2}$.

${ }^{9}$ The figures were produced by the R (2008) programming language and environment.
} 


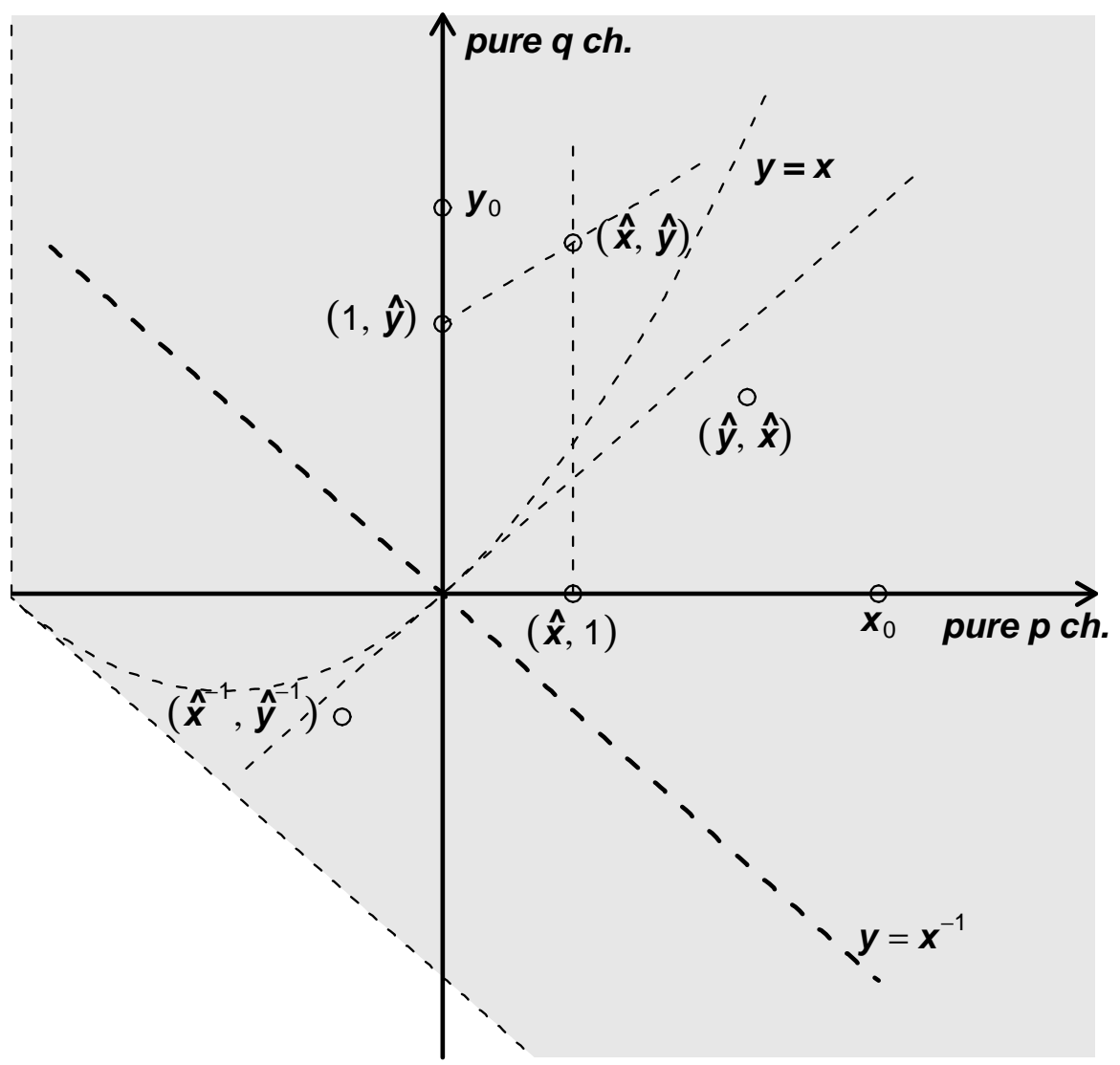

Figure 1a. The Laspeyres space

\section{$7 \quad$ Reversal tests and symmetry properties}

To illuminate further the structure of decomposition spaces we use the remaining sections to discuss the linear algebraic implications of some well-known axioms, also called tests in the index number jargon. In this section we show that the so-called reversal tests, i.e. the factor reversal and time reversal tests have basic symmetry interpretations in decomposition spaces.

First, the factor reversal test requires that the price index formula and quantity index formula should have identical functional form. In other words, switching the place of prices and quantities in a price index formula should result in the original quantity index and vice versa. In our notation this is easily seen to mean making the transformation $\left(x_{i}, y_{i}\right) \longmapsto\left(y_{i}, x_{i}\right)$ for each commodity should 


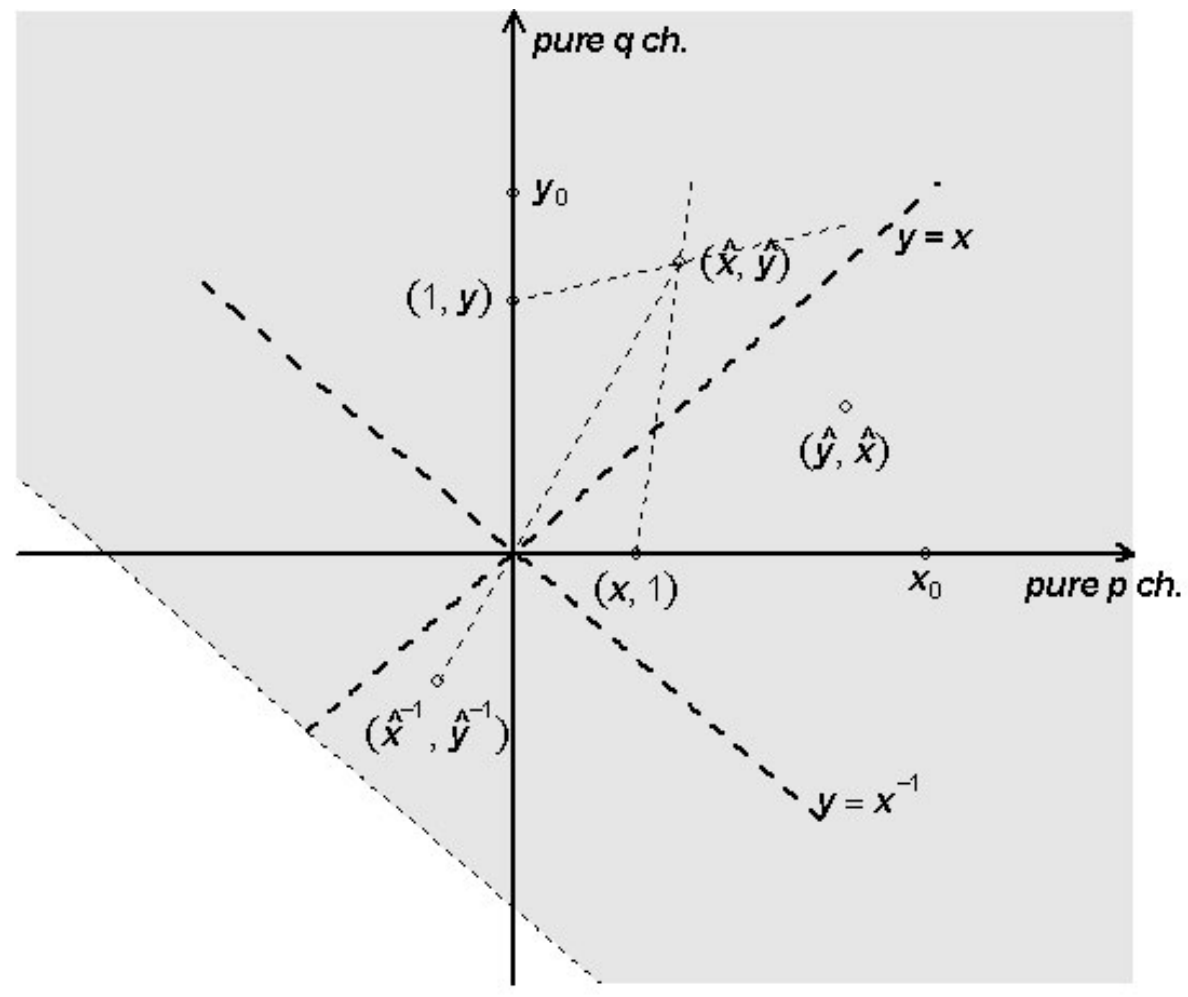

Figure 1: Figure 1b. The Stuvel space

result in the same transformation $(P, Q) \longmapsto(Q, P)$ for the index number pair.

As in the case of the identity test, we first define an analogous condition for additive decomposition functions and then show that it is equivalent to the factor reversal test. The property is called symmetry. By symmetry we mean that whenever prices and quantities are switched the price contribution and quantity contribution should also be switched, in other words we should have $d_{1}(x, y)=$ $d_{2}(y, x)$ identically.

To show equivalence of the two properties we need first to establish that factor reversibility is a linear property. Let $D$ be a decomposition space and define the factor reversal function on the convex subset $\Omega^{2} \subset D$ by setting $r(x, y)=(y, x)$. Then the factor reversal test requires that

$$
r\left(\sum_{i=1}^{n} \alpha_{i}^{0}\left(x_{i}, y_{i}\right)\right)=\sum_{i=1}^{n} \alpha_{i}^{0} r\left(x_{i}, y_{i}\right)
$$




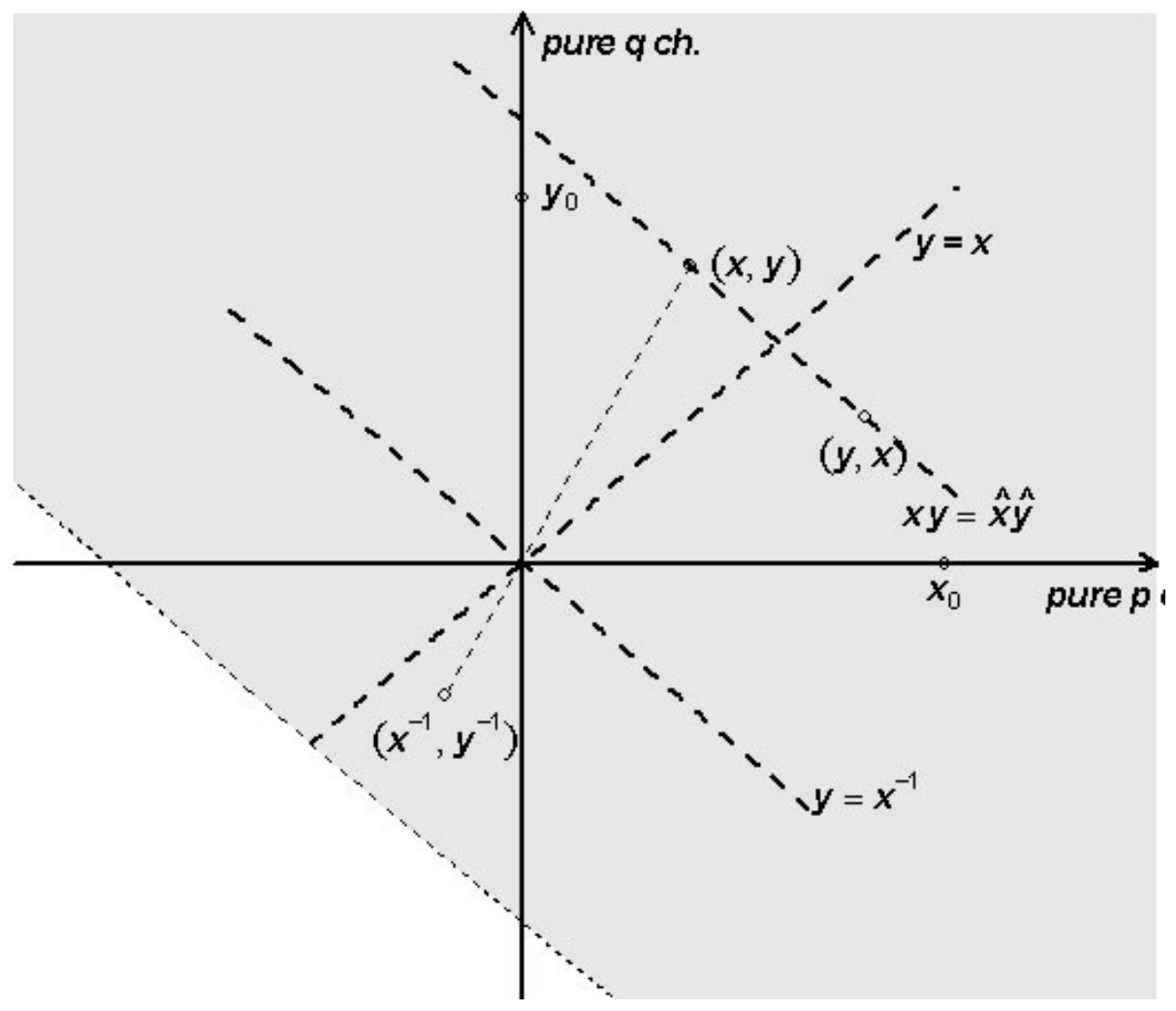

Figure 2: Fig. 1c. The Montgomery-Vartia space

where the convex combination is of course to be interpreted with respect to the operations of $D$. Clearly $r$ is bijective. Also, it follows from basic linear algebra that (5) implies that $r$ must be a restriction $r=\hat{r}_{\mid \Omega^{2}}$ of an affine bijection $\hat{r}$ : $D \rightarrow D$. As $r(1,1)=(1,1)$, so that the null decomposition is mapped to itself, $\hat{r}$ must in fact be a bijective linear map (i.e. an automorphism). Also, as $r$ it is own inverse, so that $r(r(x, y))=(x, y)$, the same is clearly true for $\hat{r}$, so that it is in fact an involution. Factor reversibility is therefore equivalent to linearity and involutiveness of the factor reversal function. It is this fact which is the main ingredient of the proof of the next equivalence theorem.

Theorem 6 A quasilinear index number pair satisfies factor reversal iff it may be represented by a symmetric additive decomposition function of expenditure change. If the index number pair is also normalized, the unique normalized additive representation is symmetric. 
Among other things Theorem 6 implies that any "rectification" procedure which produces symmetric decompositions from asymmetric ones is also a procedure for rectifying index numbers to satisfy factor reversal. For example, let $d$ be an continuous additive decomposition function and define a new additive decomposition function $\tilde{d}$ by setting $\tilde{d}_{i}(x, y)=\frac{1}{2} d_{i}(x, y)+\frac{1}{2} d_{j}(y, x)$ where $i, j=1,2$ and $i \neq j$. It is a straightforward exercise to show that the resulting function $\tilde{d}$ is a symmetric decomposition and thus defines a new index number pair which satisfies factor reversal. Also, if $d$ is already symmetric then $\tilde{d}=d$. Applying this procedure to the Laspeyres or Paasche formula pairs results in the Stuvel (1957) formula. The procedure might therefore reasonably be called Stuvel rectification to differentiate it from the better-known rectification suggested by Fisher (1922), which involves taking the geometric mean of an index number formula and its so-called factor antithesis. The two rectification procedures are not equivalent in general (and indeed the Stuvel procedure is defined only for quasilinear index numbers) but agree when either index number formula is a weighted geometric mean $^{10}$.

We call decomposition spaces that correspond to factor reversible index number pairs symmetric. Such spaces, when they are also normalized have very nice geometric properties. To see this, note that the factor reversal map $\hat{r}$ defined on a symmetric normalized space is self-adjoint with respect to the inner product structure $^{11}$. Thus its two eigenspaces are orthogonal lines in $D$. The first of these orthogonal subspaces is the span of the "diagonal" set $X=\{(x, x) \mid x \in \Omega\}$ corresponding to the case where prices and quantities have moved in exact proportion. The set $X$ is a line segment in $D$ and the corresponding eigenspace of $\hat{r}$ consists of the whole line. The second eigenspace coincides with the the kernel of the composition functional $\hat{s}$. The set of decompositions with the price and quantity changes cancelling each other out completely, that is, $K_{0}=\left\{\left(x, x^{-1}\right) \mid x \in \Omega\right\}$ is a segment of this line. Thus the inner product structure of normalized symmetric

\footnotetext{
${ }^{10} \mathrm{By}$ this we mean that the additive decomposition representation $d$ may be chosen to be of a form satisfying either $d_{1}(x, y)=w(x y) \log x$ or $d_{2}(x, y)=w(x y) \log y$ for some positive function $w$. In this case it is easy to see that the index number pair resulting from Stuvel's rectification procedure is equal to the one resulting from Fisher's rectification.

${ }^{11}$ This follows directly from the symmetry property of the additive decomposition, keeping in mind that the inner product of two decompositions in $L$ is equal to the standard inner product of the corresponding additive decompositions in $\mathbb{R}^{2}$.
} 
spaces is a natural one also in this sense: the intuitively "orthogonal" cases of perfectly proportional price and quantity change on one hand and and the case when the two completely cancel each other out are indeed orthogonal in these spaces. In addition to the pure price change and pure quantity change axes we also have an "axis of proportional change" corresponding to the subspace spanned by the set $X$ and an orthogonal axis of "no expenditure change" corresponding to $Y$. The two sets of orthogonal axes are "45 degree rotations" of each other ${ }^{12}$.

Of course, when the decomposition space $D$ is symmetric but not necessarily normalized we might use the symmetry property to establish an inner product structure in $D$ by requiring that suitable vectors in the sets $X$ and $Y$ be orthonormal. When $D$ is both normalized and symmetric the inner products produced by the two approaches are equivalent ${ }^{13}$.

We turn now to the time reversal test, which concerns the behaviour of the index number pair when direction of time is reversed, i.e. the roles of the two time periods are switched. The index number pair satisfies the test if, denoting the period 1 expenditure weights by $\alpha_{i}^{1}=\frac{v_{i}^{1}}{V^{1}}$, it satisfies $\sum_{i=1}^{n} \alpha_{i}^{1}\left(x_{i}^{-1}, y_{i}^{-1}\right)=$ $\left(P^{-1}, Q^{-1}\right)$, whenever $(P, Q)=\sum_{i=1}^{n} \alpha_{i}^{0}\left(x_{i}, y_{i}\right)$, the convex combinations of course carried out in the relevant decomposition space $D$. We may proceed similarly as in the factor reversal case. Defining the time reversal function $k$ on $\Omega^{2} \subset D$ by $k(x, y)=x y\left(x^{-1}, y^{-1}\right)$ we see that the test is in fact equivalent to the requirement that $\sum_{i=1}^{n} \alpha_{i}^{0} k\left(x_{i}, y_{i}\right)=k\left(\sum_{i=1}^{n} \alpha_{i}^{0}\left(x_{i}, y_{i}\right)\right)$. Again, the preceding equations are to be interpreted with respect to the operations of the decomposition space. By the same argument as in the case of factor reversal this implies that $k$ has to be a restriction of a linear bijection $\hat{k}: D \rightarrow D$.

\footnotetext{
${ }^{12}$ The claims are easily verified, see proof of Theorem 6 .

${ }^{13}$ However, we have decided against discussing symmetric but non-normalized spaces in this section. Such spaces are of some interest, and based on the material in this section it should be quite easy to construct the geometry of such spaces. One index number formula that is of some theoretical interest is the formula based on the decomposition given by $d_{1}(x, y)=$ $\frac{1}{2} \log \frac{x}{y}+\frac{1}{2}(x y-1)$. This could be called the geometric Stuvel index as it is a result of applying Stuvel's rectification to the geometric Laspeyres price index. In this case the Stuvel and Fisher rectifications coincide so that the resulting index number is also the geometric mean of the geometric Laspeyres index and its factor antithesis. Because of this, it satisfies factor reversal but it is equally clear that it is not normalized.
} 
In a by now familiar fashion we define an analogous condition for additive decomposition functions and show equivalence. The time symmetry condition for additive decompositions is that switching periods should result in the price and quantity contributions switching signs. More concretely, recall that the additive decomposition function decomposes expenditure change, so that $v^{1}-v^{0}=$ $v^{0} d_{1}(x, y)+v^{0} d_{2}(x, y)$. It is a natural requirement that reversing the role of periods 0 and 1 should have no effect on the absolute magnitude of the price and quantity contributions. In other words we should have $v^{1} \cdot d\left(x^{-1}, y^{-1}\right)=-v^{0} \cdot d(x, y)$. Dividing both sides by $v^{0}$, this becomes $x y \cdot d\left(x^{-1}, y^{-1}\right)=-d(x, y)$. We call an additive decomposition function $d$ time symmetric whenever it satisfies this requirement. Again, the linearity / involutiveness requirement of the time reversal function is the main ingredient of the proof for the next equivalence theorem.

Theorem 7 A quasilinear index number pair satisfies the time reversal test iff it has a time symmetric representation. In the normalized case, the unique normalized representation is time symmetric.

The geometry of the reversibility axioms can also be discussed by aid of the Figures 1a., 1b. and 1c. introduced in Section 6. Turning to the Laspeyres space in Fig. 1a. it was already mentioned that the space the set $\Omega^{2}$ is a strict subset of the half-space $H$. Also, as the Laspeyres formula is not factor reversible the space is not symmetric and $\Omega^{2}$ is not symmetric w.r.t. the 45 degree line corresponding to the "diagonal set" $X=\{(x, y) \mid y=x\}$. Also, the non-symmetry of the space may be seen in the fact that the diagonal set is not a line segment in the Laspeyres space but instead a segment of a quadratic curve. It is depicted by a thin broken line in the figure. We have also plotted a generic multiplicative decomposition $(\hat{x}, \hat{y})$ in each figure. Again, the non-symmetry of the space may be seen by noting that $(\hat{y}, \hat{x})$ is not a reflection of $(\hat{x}, \hat{y})$ with respect to the 45 degree line. The Laspeyres space is not time symmetric either, so that the decomposition $\left(\hat{x}^{-1}, \hat{y}^{-1}\right)$ is not on the straight line connecting the origin and $(\hat{x}, \hat{y})$. Note that a similar graphical representation of the decomposition space corresponding to the Paasche price - Laspeyres quantity index would be exactly the same except reflected with respect to the 45 degree line. 
In Fig. 1b. the decomposition space corresponding to the Stuvel index number pair is given. The Stuvel index is factor and time reversible which imply the corresponding symmetries for the decomposition space. First, the set $\Omega^{2}$ coincides with the half-space $H$ in $D$ and is thus symmetric w.r.t. the 45 degree line. Also, the 45 degree line coincides with the diagonal set $X=\{(x, y) \mid y=x\}$ with the decomposition $(\hat{y}, \hat{x})$ located symmetrically to $(\hat{x}, \hat{y})$ with respect to this line. The kernel of the composition functional $\hat{s}$ is identified with the line of slope -1 through the origin. The decompositions $\left(x, x^{-1}\right)$ corresponding to no expenditure change form this line. The equivalence classes $K_{w}=\{z \in D \mid \hat{s}(z)=w-1\}$ of the composition functional are identified with the lines parallel to this line, i.e. with lines of slope -1 . For $w>-1$ each line $K_{w}$ consists of all decompositions

of the expenditure change $\frac{v^{1}}{v^{0}}=w$. Time symmetry means that the line segment connecting $(\hat{x}, \hat{y})$ and $\left(\hat{x}^{-1}, \hat{y}^{-1}\right)$ passes through the origin. The kernel of the composition functional $\hat{s}$ coincides with the line $y=x^{-1}$ and divides the set $\Omega^{2}=H$ into two areas. The area below the kernel consists of all decompositions with $x y<1$, i.e. with decreased expenditure, while the area above it corresponds to the decompositions of increased expenditure, or $x y>1$. The MontgomeryVartia space depicted in Fig. 1c. shares all of the above-mentioned properties with the Stuvel space.

\section{Additional axioms and characterizations}

In this section we introduce two additional axioms and show that both of these coupled with the axioms of previous sections lead to a complete characterization of a quasilinear index number pair. First of these characterizations is a wellknown one, but we think that the linear aspect gives some new intuition to the result. We are not aware of any previous presentation of the second.

The well-known proportionality axiom proposed by Fisher (1922) requires that a price index formula should have the following property: if all price relatives are equal then the price index should be equal to the common value of the price relatives. We call this the Fisher proportionality test. The Fisher proportionality axiom may also be formulated, mutatis mutandis, for the quantity index. Again, for quasilinear index numbers this axiom has a linear algebraic 
interpretation.

Let $(P, Q)$ be a quasilinear index number pair for which both indices satisfy Fisher proportionality and let $D$ be the corresponding decomposition space. Now, as the index number pair is simply the mean in the decomposition space $D$, keeping $x$ fixed, for any convex combination of $\left(x, y_{i}\right)$ in $D$ we must have $\sum_{i=1}^{n} \alpha_{i}^{0}\left(x, y_{i}\right)$ $=(x, Q)$ for some $Q$. Thus the test is equivalent to the requirement that the sets $E_{x}=\{(x, y) \mid y \in \Omega\}$ are convex in $D$ for any fixed $x \in \Omega$. Note the similarity of this to the identity test discussed in Section 6. It is easily seen (by applying the composition functional) that in the case where $x$ is kept fixed, the quantity index $Q$ must in fact be equal to the arithmetic average of $y_{i}$ so that $Q=\sum_{i=1}^{n} \alpha_{i}^{0} y_{i}$. But once again, this implies that in fact the map $(x, y) \longmapsto y$ must be a restriction of an affine bijection from the affine hull of $E_{x}$ to $\mathbb{R}$. This in turn implies that $E_{x}$ is a one-dimensional convex set, i.e. a line segment in $D$. Similarly, the sets $F_{y}=\{(x, y) \mid x \in \Omega\}$ must be line segments. Thus for any $(x, y)$ in $\Omega^{2}$ we have $(x, y) \in E_{x} \cap F_{y}$ and the point must lie in the intersection of the two lines. It is clear that this is a very stringent requirement as we are discussing a twodimensional space. In fact, it is a known result that only a very restricted class of quasilinear formulas satisfy the Fisher proportionality test (see Balk (1996))) and the only quasilinear formula that satisfies the identity test and factor reversal test as well as Fisher proportionality is the Stuvel (1957) formula (see Table 1). The linear algebraic structure developed above allows us to re-derive this result in a very compact and informative way.

Theorem 8 If the price index of a quasilinear index number pair satisfies the identity test, the Fisher proportionality test and the factor reversal test it is the Stuvel formula.

Turning once more to the graphical representations, we see that because the Laspeyres-Paasche pair satisfies Fisher proportionality, the generic decomposition $(\hat{x}, \hat{y})$ in Fig. 1a. is seen to lie in the intersection of the line segments $E_{\hat{x}}=\{(\hat{x}, y) \mid y \in \Omega\}$ and $F_{\hat{y}}=\{(x, \hat{y}) \mid x \in \Omega\}$ which intersect the pure price and quantity change axes in points $(\hat{x}, 1)$ and $(1, \hat{y})$ respectively. A similar statement is true for Fig. 1b. corresponding to the Stuvel index The Montgomery-Vartia 
space depicted in Fig. 1c. is not exactly Fisher proportional. Therefore the sets $E_{\hat{x}}$ and $F_{\hat{y}}$ are not line segments.

One of the basic problems in the index number theory is substitution, i.e. the change in the relative importance of commodities as measured by expenditure share. As mentioned in the introduction, most index number theorists would agree that a price index formula is (in a loose sense) a generalized expenditureweighted mean of price relatives. The problem is that the expenditures on each commodity in general differ between the base and comparison periods. As prices change, there is substitution between commodities and it is not self-evident how the change in the expenditure shares should be accounted for. Consider, however, for the moment the simple case where there is no change in the expenditure shares between the two periods. In this case the index number problem is reduced to finding a suitable expenditure share -weighted mean of the price ratios. As we are dealing with calculating a mean of ratios, elementary logic seems to suggest that a prominent candidate should be the geometric mean. This leads us to define the following property: a quasilinear index number pair is a generalization of the geometric mean if the price index and quantity index reduce to the geometric expenditure-share weighted means of the price and quantity ratios respectively whenever the expenditure shares have not changed.

As the other axioms we have discussed, the generalization property may also be given a linear algebraic formulation. This is given in the proof of the next theorem, which is contained in the Appendix. Simple linear algebra shows then that the generalization axiom leads to the following characterization of the socalled Montgomery-Vartia formula given in Table 1(see e.g. Vartia (1976) and Diewert Diewert (1978) for discussion).

Theorem 9 If an index number pair is normalized and a generalization of the geometric mean it is the Montgomery-Vartia formula.

\section{Concluding remarks}

At this point it is perhaps worth noting that the preceding results do not depend on any assumptions except quasilinearity. If, as would seem natural considering 
the subject matter, we are willing to impose the requirement that index number formulas be continuous, then the next corollaries follow immediately from Theorems 3, 5, 6 and 7 ..

Corollary 1 The following are equivalent:

1. An index number pair $(P, Q)$ is (i) consistent in aggregation, (ii) continuous, (iii) one-to-one and (iv) weakly proportional.

2. There exists a continuous additive decomposition function of value change $d$ so that $(P, Q)$ is the d-mean.

Corollary 2 An index number pair $(P, Q)$ is quasilinear with a unique continuous normalized decomposition representation iff it satisfies the identity test as well as (i)-(iv), Similarly, the pair $(P, Q)$ is quasilinear with a continuous (time) symmetric additive representation iff in addition to (i)-(iv) it satisfies the factor (time) reversal test.

All of the formulas in Table 1 are continuous. Some of their other properties are listed in Table 2 as an example ${ }^{14}$. For the normalized formulas, Laspeyres, Paasche, Stuvel and Montgomery-Vartia, the additive decomposition given in Table 1 in fact gives the unique normalized representation ${ }^{15}$.

The results derived so far are in our opinion quite remarkable. They imply that the quasilinear index number problem is in fact the same problem as finding an additive decomposition of expenditure change, a much less-studied problem (see for example Balk (2003) and Diewert (2005)). Quasilinear index numbers and additive measures of expenditure change are simply different representations of the same aggregation problem. The additive decomposition function is given by the coordinates of the index number pair with respect to a basis in a certain linear space representation. Also, the sense in which the two decompositions are

\footnotetext{
${ }^{14}$ Again, the list of formulas is in no way exclusive. Also, while only certain combinations of the three properties occur in the table, it is easy to come up with quasilinear formulas with any combination of these.

${ }^{15}$ For non-normalized ones we have given the unique representation which is approximately normalized in the sense that the linear Taylor approximation around the null decomposition $(1,1)$ is normalized.
} 


\begin{tabular}{|l|l|l|l|}
\hline Formula & Normalized & Symmetric & Time symmetric \\
\hline Laspeyres & Yes & No & No \\
Paasche & Yes & No & No \\
Geometric (log-)Laspeyres & No & No & No \\
Geometric (log-)Paasche & No & No & No \\
Stuvel & Yes & Yes & Yes \\
Montgomery-Vartia & Yes & Yes & Yes \\
\hline
\end{tabular}

Table 2: Properties of additive decompositions corresponding to well-known formulas

identical is mathematically straightforward, for example, Fourier series representations of functions are identical with their standard representations in exactly the same sense. Of course, the structure here is much simpler, but the analytical benefits are the same: some things are easier to study in one representation than in the other.

A direct implication of this result is that an axiomatization of index numbers implies an axiomatization for additive decomposition functions which is its exact isomorphic counterpart. The converse of course also holds. Thus any axiom which does not make sense in both representations may be considered suspect. Also, as a lesser point, while the connection between additive and multiplicative decompositions have been discussed in the literature (see again for example Balk (2003) and Diewert (2005)) the fact that there is an exact sense in which an additive decomposition function corresponds to a quasilinear index number has not been noticed.

The results also suggest that consistency in aggregation or quasilinearity is a natural property of index number formulas. In addition to being intuitively appealing, consistency in aggregation may in fact be interpreted as consistency between the treatment of differences and relative changes. This is an important property which is lost when index number formulas with more complex aggregative structure are used. This is in our opinion as sound an argument for the adoption of these indices as may be expected from the axiomatic strand of index number theory. 


\section{References}

ACZÉL, J - HOSSZÙ, M. (1956): On Transformations with Several Variables and Operations in Multidimensional Spaces. Acta Mathematica Acad. Sci. Hungaricae, 7, 327-338.

ACZÉL, J. (1966): Lectures on Functional Equations and their Applications. Academic Press, New York.

BALK, B. M.(1995): Axiomatic Price Index Theory: A Survey. International Statistical Review, 63:69-93.

- (1996): Consistency-in-Aggregation and Stuvel Indices. Review of Income and Wealth 42: 353-363.

- (2003): Ideal Indices and Indicators for Two or More Factors. Journal of Economic and Social Measurement 28: 203-217.

BLACKORBY, C., DONALDSON, D. (1984): Social Criteria for Evaluating Population Change. Journal of Public Economics 25: 13-33.

BLACKORBY, C., PRIMONT, D., RUSSELL, R.R. (1978): Duality, Separability, and Functional Structure: Theory and Economic Applications. North Holland, New York.

BLACKORBY, C., PRIMONT, D. (1980): Index Numbers and Consistency in Aggregation. Journal of Economic Theory 22: 87-98.

DIEWERT, W. E. (1978): Superlative Index Numbers and Consistency in Aggregation. Econometrica, 46: 883-900.

- (1993): Essays on Index Numbers. North Holland.

- (2005): Index Number Theory Using Differences Rather Than Ratios. American Journal of Economics and Sociology, 64: 311-360.

FISHER, I. (1922): The Making of Index Numbers. Houghton Mifflin Company.

GORMAN, W.M.(1986): Compatible Indices. Economic Journal, Supplement: Conference papers 96: 83-95.

GRILLET, P. A. (2001): Commutative Semigroups. Kluwer Academic Publishers. Dordrecht.

KOLMOGOROV, A.I.(1930): Sur la notion de la moyenne. Atti della R. Accademia Nazionale dei Lincei 12, 388-391.

LAWSON, J., MADISON, B. (1971): On Congruences and Cones. Mathematische Zeitschrift, $120,18-24$

LAWSON, J. (1990): Embedding Semigroups in Lie Groups. In Hoffman, L. Hoffman, P. and Hoffman (eds.), K:Analytical and Topological Theory of Semigroups: Trends and Developments. Walter de Gruyter.

NAGUMO (1930): Über eine Klasse der Mittelwerde. Japanese Journal of Mathematics 7: 7179 .

PURSIAINEN, H. (2007): Consistency in aggregation, quasilinear means and index numbers. A working paper. 
R DEVELOPMENT CORE TEAM (2008). R: A language and environment for statistical computing. R Foundation for Statistical Computing, Vienna, Austria. ISBN 3-900051-07-0, URL http://www.R-project.org.

RÅDSTRÖM, H. (1952): An Embedding Theorem for Spaces of Convex Sets, Proc. Am. Math. Soc. 3: $165-169$.

ROTHMAN, N. J. (1960): Embedding of Topological Semigroups. Math. Annalen 139: 197203.

SAMUELSON, P. A.- SWAMY S. (1974): Invariant Economic Index Numbers and Canonical Duality: Survey and Synthesis. The American Economic Review 64: 566-593.

STUVEL, G. (1957): A New Index Number Formula. Econometrica 25, 123-131.

- (1989): The Index Number Problem and Its Solution. Macmillan Press Ltd., London.

How Should Relative Changes Be Measured? The American Statistician, 39: 43-46.

VARTIA, Y. O. (1976): Relative Changes and Index Numbers. Research Institute for the Finnish Economy, Series A.

\section{A Proofs of theorems}

Proof of Theorems 1 on pg. $\mathbf{5}$ and Theorem $\mathbf{2}$ on pg. 6. Theorems 1 and 2 are separated in the text for readability, but it is convenient to give a combined proof. To prove that any quasilinear mean is the mean of some space $L$ we in fact prove that the $f$-extension of point 4 of Theorem 2 always exists. Let $f: A \rightarrow C \subset \mathbb{R}^{k}$ define a quasilinear mean.

First, note that to prove the existence of an $f$-extension we only need to construct a set $L$ and a bijection $\widehat{f}: L \rightarrow \mathbb{R}^{k}$ such that $A \subset L$ and $f=\widehat{f}_{\mid A}$. This is because if such an $\widehat{f}$ exists we may define scalar multiplication on $L$ by setting $\alpha z=\hat{f}^{-1}(\alpha \hat{f}(z))$ for any $\alpha \in \mathbb{R}$ and $z \in L$. Similarly, vector addition in $L$ may be defined simply by $z+z^{\prime}=\hat{f}^{-1}\left(\hat{f}(z)+\hat{f}\left(z^{\prime}\right)\right)$ for any $z, z^{\prime} \in L$. It is clear that these operations satisfy the axioms of a linear space. The function $\hat{f}$ is by construction a linear bijection from $L \rightarrow \mathbb{R}^{k}$. Thus, as the inverse image of a full-dimensional convex set $C, A$ must also be a full-dimensional convex set. It also follows that $\operatorname{dim}(L)=\dot{k}$, so that point 1 of Theorem 2 also follows. Now, if $x, y \in A \subset$ $L$, then $\hat{f}(x)=f(x) \in C$ and $\hat{f}(y)=f(y) \in C$. For any convex combination of $x, y$ in $A$ we have $\alpha x+(1-\alpha) y=\hat{f}^{-1}(\alpha \hat{f}(x)+(1-\alpha) \hat{f}(y))=\hat{f}^{-1}(\alpha f(x)+(1-\alpha) f(y))=$ $f^{-1}(\alpha f(x)+(1-\alpha) f(y))$, where the last step follows from convexity of $C$. Thus, taking convex combinations in the subset $A$ of the linear space $L$ coincides with the quasilinear mean representable by $f$.

Thus to prove the existence of a $k$-dimensional $f$-extension we only have to construct the function $\widehat{f}$. Assume first that the origin is contained in $C$, i.e. $0 \in C$. Let $K=\mathbb{R} \times A \times$ $A$. Define the following binary relation $R$ in $K:(\alpha, x, y) R\left(\alpha^{\prime}, x^{\prime}, y^{\prime}\right)$ iff $\alpha[f(x)-f(y)]=$ $\alpha^{\prime}\left[f\left(x^{\prime}\right)-f\left(y^{\prime}\right)\right]$. It is straightforward to show that $R$ is an equivalence. Let $L=K \mid R$ be the set of equivalence classes of $R$ and let $p: K \rightarrow L$ be the function mapping each element of $K$ to its equivalence class. Also, for each $x \in A$ define $r(x)=p\left(1, x, f^{-1}(0)\right)$. This is a one-to-one map which maps $A$ to $A^{\prime}=r(A)$. Let $\widehat{f}$ be the bijective function defined by $\widehat{f}[p(\alpha, x, y)]=\alpha[f(x)-f(y)]$. Clearly, as $C$ was assumed to be $k$-dimensional, $\widehat{f}(L)=\mathbb{R}^{k}$ and $\widehat{f}(r(x))=1 \cdot(f(x)-0)=f(x)$ for $x \in A$. This implies that $r$ is a bijective function between $A$ and $A^{\prime}$ and we can equate the two sets by equating each $x \in A$ with its image 
$x^{\prime}=r(x) \in A^{\prime}$ and regard $A$ as a subset of $L$. Thus, $L$ endowed with the appropriate scalar multiplication and vector addition operations is an $f$-extension of $A$.

Actually, the assumption $0 \in C$ does not imply loss of generality. This is because if $0 \notin C$, we may consider the map $g$ defined by $g(x)=f(x)-y_{0}$ where $y_{0}$ is a constant $y_{0} \in C$ instead of $f$. It is a matter of simple calculation to verify that it defines the same quasilinear mean as $f$. Then the set $C^{\prime}=g(A)=C-y_{0}$ is convex and $0 \in C^{\prime}$. By the previous argument, it is possible to construct a $g$-extension $L^{\prime}$ of $A$. Let $\widehat{g}$ be the corresponding extension of $g$. Define the function $\widehat{f}$, given by $\widehat{f}(x)=\widehat{g}(x)+y_{0}$. Clearly $\widehat{f}$ is the function required to construct the $f$-extension.

Thus we have proved the existence of an $f$-extension and by implication the first part of Theorem 1. The converse is almost trivial. If $L$ is an extension of $A$ it is by definition a finite-dimensional linear space containing $A$ as a full-dimensional convex subset. Let $\operatorname{dim}(L)=k$. Elementary linear algebra implies that the coordinate function corresponding to any basis of $L$ is a linear bijection $\hat{f}: L \rightarrow \mathbb{R}^{k}$. Let $f$ be the restriction $f=\hat{f}_{\mid A}$. Then by linearity and convexity of $A$ we have for any convex combination of $x, y \in A$ : $\alpha x+(1-\alpha) y=\hat{f}^{-1}(\alpha \hat{f}(x)+(1-\alpha) \hat{f}(y))=f^{-1}(\alpha f(x)+(1-\alpha) f(y))$ and thus convex combinations coincide with some quasilinear mean on $A$. Thus Theorem 1 holds.

In the proof of Theorem 1 we have also proved points 1 and 4 of Theorem 2 . Turning to point 2 it was already mentioned that simple calculation is all that is needed to verify the if part. For the only if part, note that if $g$ represents the same mean as $f$, we have already proved the existence of $f$ - and $g$-extensions $L$ and $L^{\prime}$ as well as the extended linear bijections $\hat{f}: L \rightarrow \mathbb{R}^{k}$ and $\hat{g}: L^{\prime} \rightarrow \mathbb{R}^{k}$. The set $A$ is a convex full-dimensional subset of both spaces and any convex combinations of elements of $A$ coincide in the two spaces. Thus the map $I: A \subset L \rightarrow A \subset L^{\prime}$ defined by $I(x)=x$ is a map from a full-dimensional convex subset of $L$ to $L^{\prime}$, which preserves convex combinations. By elementary linear algebra it follows that $I$ must be a restriction of an affine bijection $\hat{I}: L \rightarrow L^{\prime}$. Thus $\hat{a}=\hat{g} \circ \hat{I} \circ \hat{f}^{-1}: \mathbb{R}^{k} \rightarrow \mathbb{R}^{k}$ is affine as a composition of affine and linear functions and looking at the restriction $a=\left.\hat{a}\right|_{C}$ we see that $a(f(x))=g(x)$ for any $x$ in $A$ so that indeed $g$ is an affine transformation of $f$. Also, the existence of the map $\hat{I}$ implies that point 3 of the theorem is true.

Turning to the point 5 the function $\hat{f}: L \rightarrow \mathbb{R}^{k}$ is a linear bijection, i.e. an isomorphism of $L$ and $\mathbb{R}^{k}$ and thus by elementary linear algebra it is the coordinate function for the basis $U$ consisting of vectors $u_{1}, \ldots, u_{k}$ which are given by the equations $\hat{f}\left(u_{i}\right)=e_{i}$ for $i \in\{1, . ., k\}$ where $e_{i} \in \mathbb{R}^{k}$ is the $i$ th canonical basis vector. Also, clearly $\hat{f}(x)=0$ iff $x=0_{L}$, i.e. the origin of $L$. Thus $5 \mathrm{a}$ and $5 \mathrm{~b}$ follow from the construction of $\hat{f}$. It follows directly from the above discussion that if $g$ is an affine transformation of $f$ then the corresponding spaces are affine transformations of each other. If $g$ is a linear transformation of $f$, then it is clear that $\hat{g}$ is a bijective linear transformation of $\hat{f}$ so that the two are simply coordinate functions for two different bases of $L$.

Formal definition of consistency in aggregation on pg. 11. Consistency in aggregation can be formalized as follows. A permutation $\left(x_{1}^{\prime}, \ldots, x_{n}^{\prime}\right)$ of an $n$-tuple $\left(x_{1}, \ldots, x_{n}\right)$ of elements $x_{i}$ of some set $A$ is obtained by putting $x_{i}^{\prime}=x_{j(i)}$, where $j$ is any bijection from the set $\{1, \ldots, n\}$ to itself. It is also always possible to partition an $n$-tuple into $K$ non-empty $n_{k}$-tuples $\left(x_{k, 1}, \ldots, x_{k, n_{k}}\right)$, with $k=1, \ldots, K, 1 \leq K \leq n$ and $n_{1}+\ldots+n_{K}=n$. Now, let $\left(P_{k}^{\prime}, Q_{k}^{\prime}\right)=h_{K}\left(\left(x_{k, 1}^{\prime}, y_{k, 1}^{\prime}, v_{k, 1}^{\prime 0}\right), \ldots,\left(x_{k, n_{k}}^{\prime}, y_{k, n_{k}}^{\prime}, v_{k, n_{k}}^{\prime 0}\right)\right)$ be the $k$ th sub-index pair corresponding to an arbitrary partition of an arbitrary permutation of the data and let $V_{k}^{\prime}=\sum_{i=1}^{n_{k}} v_{k, i}^{\prime 0}$ be the corresponding period 0 expenditure aggregate. Also, let $(P, Q)$ be the value of the index number pair calculated directly from the data. Then the index number formula pair is consistent in aggregation iff

$$
(P, Q)=h_{K}\left(\left(P_{1}^{\prime}, Q_{1}^{\prime}, V_{1}^{\prime}\right), \ldots,\left(P_{K}^{\prime}, Q_{K}^{\prime}, V_{K}^{\prime}\right)\right) .
$$


As mentioned, it is straightforward to show that the quasilinear mean index number pairs satisfy this. However, it is a non-trivial task to show that this property also "almost" implies the quasilinear mean form.

Proof of Lemma 1 on pg. 12. Let $(P, Q)$ be a quasilinear index number pair, i.e. satisfying equations (1) and (2) for some function $d$. By point 3 of Theorem 2 we may replace $d$, when necessary by any affine transformation of it. Certainly there are affine transformations that map $(1,1)$ to the origin. Thus, making the replacement if necessary, we may always assume $d(1,1)=0 \in \mathbb{R}^{2}$.

Consider now the two-dimensional $d$-extension $D$ of $\Omega^{2}$ the existence of which is guaranteed by the same theorem. By point $5 D$ has origin $(1,1)$. Let $\hat{d}: L \rightarrow \mathbb{R}^{2}$ be the linear extension of $d$. By construction, the mean of the space $D$ coincides with the index number pair $(P, Q)$ on the convex subset $\Omega^{2}$. Consider now the function $t: \Omega^{2} \rightarrow \Omega$ defined by $t(x, y)=x y$. We may interpret this as mapping the convex subset $\Omega^{2}$ of the linear space $D$ to the convex subset $\Omega$ of the one-dimensional Euclidean space $\mathbb{R}$. Note also the obvious fact that the standard $\alpha_{i}^{0}$-weighted arithmetic mean of the expenditure ratios $\frac{v_{i}^{1}}{v_{i}^{0}}=x_{i} y_{i}$ is the aggregate expenditure ratio, or $\sum_{i=1}^{n} \alpha_{i}^{0}\left(\frac{v_{i}^{1}}{v_{i}^{0}}\right)=\frac{V^{1}}{V^{0}}$. Using the function $t$ above we see that this may be written as $\sum_{i=1}^{n} \alpha_{i}^{0} t\left(x_{i}, y_{i}\right)=\frac{V^{1}}{V^{0}}$. As $(P, Q)$ is an index number pair it satisfies (2), which may be rewritten, using the function $t$ as $t(P, Q)=\frac{V^{1}}{V^{0}}$. Combining the above equations we see that we must have

$$
t\left(\sum_{i=1}^{n} \alpha_{i}^{0}\left(x_{i}, y_{i}\right)\right)=\sum_{i=1}^{n} \alpha_{i}^{0} t\left(x_{i}, y_{i}\right)
$$

But as we have noted before, basic linear algebra implies that this is satisfied only if $t$ is a restriction of some affine map $\hat{t}: D \rightarrow \mathbb{R}$. Taking the value of $\hat{t}$ at the origin we have $\hat{t}((1,1))=1 \cdot 1=1$, which implies that $\hat{s}$ defined by $\hat{s}(z)=z-1$ is a linear functional. Thus, $D$ is a decomposition space.

Now, assume that $D$ is a decomposition space. By Theorem 1 convex combinations on the convex subset $\Omega^{2}$ coincide a quasilinear mean on $\Omega^{2}$. Let $\hat{s}: D \rightarrow \mathbb{R}$ be the composition functional and let $s=\hat{s}_{\mid \Omega^{2}}$. Clearly, $P Q-1=s\left(\sum_{i=1}^{n} \alpha_{i}^{0}\left(x_{i}, y_{i}\right)\right)=\sum_{i=1}^{n} \alpha_{i}^{0} s\left(x_{i}, y_{i}\right)=\frac{V^{1}}{V^{0}}-1$ so that (2) is also satisfied.

Proof of Theorem 4 on pg. 13. Let $d$ define an index number pair on $\Omega^{2}$, let $D$ be the corresponding decomposition space and let $\hat{d}: D \rightarrow \mathbb{R}^{2}$ be the linear extension of $d$. We have already established that this implies that there must exist a linear functional $\hat{s}$ with $\hat{s}(x, y)=$ $x y-1$ for $(x, y) \in \Omega^{2} \subset D$. Consider now the function $l: \mathbb{R}^{2} \rightarrow \mathbb{R}$, defined by $l=\hat{s} \circ \hat{d}^{-1}$ is linear as a composite of two linear functions. It is associated with matrix $B=\left[\begin{array}{ll}a & b\end{array}\right]$ (with either $a$ or $b$ or both non-zero). By definition, $l(d(x, y))=a d_{1}(x, y)+b d_{2}(x, y)=\hat{s}(x, y)=x y-1$. Any linear transformation of $d$ represents the same index number pair and also results in the same decomposition space $D$. This holds in particular the linear transformation $\tilde{d}$ consisting of components $\tilde{d}_{1}(x, y)=\frac{1}{2}(a+b) d_{1}(x, y)+\frac{1}{2}(b-a) d_{2}(x, y)$ and $\tilde{d}_{2}(x, y)=\frac{1}{2}(a-b) d_{1}(x, y)+$ $\frac{1}{2}(a+b) d_{2}(x, y)$. It is straightforward to check that $\tilde{d}_{1}(x, y)+\tilde{d}_{2}(x, y)=x y-1$.

Conversely, assume $d_{1}(x, y)+d_{2}(x, y)=x y-1$ and let $D$ be the $d$-extension of $\Omega^{2}$.The equation may be written as $C d(x, y)=s(x, y)=x y-1$ where $C$ is the matrix $C=\left[\begin{array}{ll}1 & 1\end{array}\right]$. Let $\hat{d}: \mathbb{R}^{2} \rightarrow \mathbb{R}$ be the linear extension of $d$. Then the function $\hat{s}$ defined by $\hat{s}=C \hat{d}$ is a linear functional on $D$ (it is linear as it is a composite of linear functions). Clearly $\hat{s}$ coincides with $s$ on $\Omega^{2}$ and thus $D$ is a decomposition space.

Proof of Theorem 5 on pg. 16. Let now $d$ define an index number pair and let $D$ be the corresponding decomposition space. It was already seen that an index number pair satisfies the identity tests iff the sets $E_{1}=\{(x, 1) \mid x \in \Omega\}, E_{2}=\{(1, y) \mid y \in \Omega\}$ are convex in $D$.Assume 
that the test is satisfied. We now prove that $E_{1}$ and $E_{2}$ are linearly independent. By the identity test, $\sum_{i=1}^{n} \alpha_{i}^{0}\left(x_{i}, 1\right)=(P, 1)$ and $\sum_{i=1}^{n} \alpha_{i}^{0}\left(1, y_{i}\right)=(1, Q)$ always. Applying the composition functional $\hat{t}$ on both sides of the equation $\sum_{i=1}^{n} \alpha_{i}^{0}\left(x_{i}, 1\right)=(P, 1)$ shows that $P=\sum_{i=1}^{n} \alpha_{i}^{0} x_{i}$. But this implies that the map $E_{1} \rightarrow \mathbb{R}$ given by $(x, 1) \longmapsto x$ must be a restriction of an affine bijection which clearly implies that the linear span $L_{1}$ of $E_{1}$ is a one-dimensional linear space, i.e. a straight line through the origin in $D$. By similar reasoning, the set $E_{2}$ must also be a segment of a line through the origin. Thus, if the two sets would lie on the same line we would have to have $(x, 1)=\alpha(1, x)$ for some $x \neq 1$. Applying the composition functional on each side gives the equation $x=\alpha x$ which shows that we would have to have $\alpha=1$ which is clearly a contradiction. Thus the sets are linearly independent and $D$ may be represented as a direct sum $D=L_{1} \oplus L_{2}$.

Let now $x_{0}=(2,1) \in L_{1}$ and $y_{0}=(1,2) \in L_{2}$. The two vectors form a basis of $D$. Let $\hat{d}$ be the coordinate map mapping each $z$ to its $x_{0}, y_{0}$-coordinates. Then the restriction $d=\left.\hat{d}\right|_{\Omega^{2}}$ is a representation of the index number pair under consideration. Any decomposition $(x, y) \in \Omega^{2}$ may thus be uniquely represented as $(x, y)=\lambda x_{0}+\mu y_{0}$ where $d_{1}(x, y)=\lambda$ and $d_{2}(x, y)=\mu$. Thus, applying the composition functional on this equation, we have $x y-1=$ $\hat{s}(x, y)=\lambda \hat{s}\left(x_{0}\right)+\mu \hat{s}\left(y_{0}\right)=\lambda+\mu$ so that $d$ is indeed an additive decomposition function. Also, by definition $d_{1}(x, 1)=d_{2}(1, y)=0$ so that $d$ is normalized.

Assume now that an index number pair may be represented by a normalized decomposition $d$. Then $d$ is the restriction $d=\left.\hat{d}\right|_{\Omega^{2}}$ of a coordinate function for some basis $x_{0}, y_{0}$ of $D$. Noting that $d_{1}(2,1)=1$ and $d_{2}(2,1)=0$ it is clear that $x_{0}=(2,1)$. Similarly, it must be that $y_{0}=(1,2)$. Let $L_{1}$ be the subspace spanned by $x_{0}$ and let $L_{2}$ be the subspace spanned by $y_{0}$. As $d$ is normalized, clearly $E_{1} \subset L_{1}$ and $E_{2} \subset L_{2}$. Also, the restriction of the composition functional $\left.\hat{s}\right|_{L_{1}}: L_{1} \rightarrow \mathbb{R}$ is a bijective linear map. The image $\hat{s}\left(E_{1}\right)=\Omega-\{1\}$ is a convex subset of $\mathbb{R}$. As convexity is preserved under linear transformations, $E_{1}$ is convex. Similarly, $E_{2}$ may be shown to be convex and thus the identity test is satisfied.

Proof of Theorem 6 on pg. 21. Assume that a quasilinear index number pair represented by the additive decomposition function $d$ satisfies factor reversal. We saw that the factor reversal function $r$ must then be a restriction of a bijective linear map $\hat{r}: D \rightarrow D$. Let $C$ be the matrix corresponding to $\hat{r}$ in the basis implied by $d$, i.e. given by $C \hat{d}(z)=\hat{d}(\hat{r}(z))$. Noting that $(x, y)=r(r(x, y))$ we see that $\hat{r}(\hat{r}(z))=z$ which means that $\hat{r}^{-1}=\hat{r}$. Thus, also $C C=I$ and examining the Jordan form of $C$ immediately yields the result (a basic property of involutions) that $C$ must be diagonalizable with eigenvalues either $\lambda=1$ or $\lambda=-1$. Clearly at least one eigenvalue must have value $\lambda=-1$ as $\hat{r}$ cannot be the identity transformation. Also, both eigenvalues of $C$ which are also the eigenvalues of $\hat{r}$ cannot be equal to -1 . This is because the subspace spanned by the "diagonal" set $X=\{(x, x) \mid x \in \Omega\}$ must be at least one-dimensional in $D$ and as $r(x, x)=(x, x)$ it is contained in the eigenspace corresponding to the eigenvalue 1 . We conclude that $C$ and thus $\hat{r}$ are diagonalizable with eigenvalues $\lambda_{1}=1$ and $\lambda_{2}=-1$. Let now $u, v \in D$ be a basis in which $\hat{r}$ is diagonal with $u, v$ eigenvectors corresponding to eigenvalues $\lambda_{1}=1$ and $\lambda_{2}=-1$ respectively. Switching prices and quantities leaves the product of the relatives unchanged, and thus $\hat{r}$ must also leave the value of the composition functional unchanged. Thus, $\hat{s}(\hat{r}(v))=-\hat{s}(v)=\hat{s}(v)$ and we must have $\hat{s}(v)=0$ so that the eigenspace corresponding to the negative eigenvalue is in fact the kernel of the composition functional. Therefore is is clearly possible to choose $u$ such that $\hat{s}(u)=1$ in which case for any vector $z=\alpha u+\beta v$ we have $\alpha=\hat{s}(z)$. In particular, for any decomposition $(x, y)=\alpha u+\beta v$ the coefficient $\alpha$ must be $\alpha=x y-1$. Let $j$ be the restriction to $\Omega^{2}$ of the coordinate function for the basis $u, v$ and define $d(x, y)=\frac{1}{2}\left[\begin{array}{cc}1 & 1 \\ 1 & -1\end{array}\right] j(x, y)=A j(x, y)$. As a linear transformation of $j$, $d$ represents the same index number pair. Also, $d_{1}(x, y)+d_{2}(x, y)=j_{1}(x, y)=x y-1$ so that $d$ is an additive decomposition function. Also, clearly $d(y, x)=A\left[\begin{array}{cc}1 & 0 \\ 0 & -1\end{array}\right] A^{-1} d(x, y)=$ $\left[\begin{array}{ll}0 & 1 \\ 1 & 0\end{array}\right] d(x, y)$ so that it is symmetric. 
Assume conversely that $(P, Q)$ has a symmetric decomposition representation $d$. Let $u, v$ be the basis corresponding to $d$. Then for any $(x, y)=\alpha u+\beta v$ we have $r(x, y)=\beta u+\alpha v$ so that $r$ is a restriction of a linear map and $(P, Q)$ satisfies factor reversal.

It remains to show that for normalized index number pairs that satisfy factor reversal the normalized decomposition representation is symmetric. Let $d$ be such a representation and let $x_{0}=(2,1), y_{0}=(1,2)$ be the orthonormal basis corresponding to $d$. Then the vectors $u=\left(x_{0}+y_{0}\right), v=\left(x_{0}-y_{0}\right)$ are also orthogonal and it is easy to see that they form a basis in which $\hat{r}$ is diagonal with $u, v$ eigenvectors for eigenvalues $\lambda_{1}=1$ and $\lambda_{2}=-1$ respectively. Also, $x_{0}=\frac{1}{2}(u+v), y_{0}=\frac{1}{2}(u-v)$ so that $\hat{r}\left(x_{0}\right)=y_{0}$ and $\hat{r}\left(y_{0}\right)=x_{0}$. But this means that $d$ is symmetric.

Proof of Theorem 7 on pg. 24. Let $D$ be the decomposition space of an index number pair satisfying time reversal and let $\hat{k}$ be the linear extension of the time reversal function defined above. We have to show that $\hat{k}(z)=-z$ for all $z$. It is easy to see that as in the case of the factor reversal map we have $\hat{k}=\hat{k}^{-1}$. Again using an argument similar to the factor reversal case it is easy to show that this implies that $\hat{k}$ is diagonalizable with eigenvalues either 1 or -1 . Both eigenvalues cannot be unity as $\hat{k}$ is not the identity transformation. This can be seen by noting that for any decomposition $(x, y)$ we have $x^{-1} y^{-1}-1=-\frac{1}{x y}(x y-1)$ which implies that $\hat{s}(\hat{k}(z))=-\hat{s}(z)$. Thus at least one of the eigenvalues is negative. Assume that the other is positive. Then there exists a one-dimensional eigenspace spanned by some vector $u$ such that $\hat{k}(\alpha u)=\alpha u$ for all $\alpha$. But using the fact that $\hat{s}(\hat{k}(u))=-\hat{s}(u)$ we see that $-\hat{s}(u)=\hat{s}(u)$ and thus the eigenspace corresponding to eigenvalue $\lambda=1$ coincides with $\operatorname{Ker}(\hat{s})$. But then we would have $\hat{k}\left(x, x^{-1}\right)=\left(x, x^{-1}\right)$ for any $x \in \Omega$. As $\hat{k}\left(x, x^{-1}\right)=\left(x^{-1}, x\right)$ this is a contradiction and therefore both eigenvalues are equal to -1 which implies that $\hat{k}(z)=-z$ for all $z$.

Conversely, assume that there exists a time symmetric additive decomposition representation $d$ for a quasilinear index number pair. Let $u, v$ be the basis corresponding to $d$. Then for $(x, y)=\alpha u+\beta v$ we have $k(x, y)=-\alpha u-\beta v$ so that $k$ is indeed the restriction of a linear map.

It is clear that any decomposition representation of a time reversible quasilinear index number pair is time symmetric, thus in particular the unique normalized representation is time symmetric when it exists.

Proof of Theorem 8 on pg. 26. It was noted above that each set $E_{x}$ and $F_{y}$ is a line segment and that the point $(x, y)$ lies in the intersection of the two lines. Also, it was seen that the function $(x, y) \longmapsto x$ from $F_{y}$ to $\mathbb{R}$ is a restriction of an affine bijection for fixed $y$. Thus, using the fact that the representation $d$ of the index number formula pair must be a restriction of a linear map we see that necessarily $d(x, y)=\left[\begin{array}{l}a_{1}(y) \\ a_{2}(y)\end{array}\right] x+\left[\begin{array}{l}b_{1}(y) \\ b_{2}(y)\end{array}\right]$. But remembering that the same thing must hold for the function $(x, y) \longmapsto y$ for fixed $x$, we also see that $d(x, y)=\left[\begin{array}{l}c_{1}(x) \\ c_{2}(x)\end{array}\right] y+\left[\begin{array}{l}d_{1}(x) \\ d_{2}(x)\end{array}\right]$. Thus $d_{1}(x, y)=a_{1}(y) x+b_{1}(y)=c_{1}(x) y+d_{1}(x)$. As normalization was assumed we can assume $d_{1}(x, 1)=x-1$ and $d_{1}(1, y)=0$. Using these it is easy to see it must hold that $a_{1}(y)=-b_{1}(y), d_{1}(x)=x-1-c_{1}(x)$, so that $\left(a_{1}(y)-1\right)(x-1)=c_{1}(x)(y-1)$ which in turn is possible only if $a_{1}(y)=\alpha(y-1)+1$ and $c_{1}(x)=\alpha(x-1)$ for some constant $\alpha$. Thus $d_{1}(x, y)=\alpha(y-1)(x-1)+(x-1)$. By symmetry we may assume that $d_{2}(x, y)=d_{1}(y, x)$ which is possible only for $\alpha=\frac{1}{2}$. This

results in the Stuvel formula. $\mathbf{~}$ Proof of Theorem $\mathbf{9}$ on $\mathbf{~ p g}$. 27.. First, note that expenditure shares remain unchanged if and only if all expenditures change proportionally by some common factor $w$ so that $x_{i} y_{i}=$ $w$ for all $i$. Using the composition functional the expenditure shares remain unchanged iff $\hat{s}\left(x_{i}, y_{i}\right)=w-1$ for all $i$. Now, let the sets $K_{w}=\{z \in D \mid \hat{s}(z)=w-1\}$ be the equivalence classes defined by $\hat{s}$. As $\hat{s}$ is linear, each of these sets is a line in $D$, more precisely each is a line parallel to the kernel $K_{1}=\operatorname{Ker}(\hat{s})$ of the composition functional. (The set of these equivalence classes actually define the quotient space $D \mid \operatorname{Ker}(\hat{s}))$. Therefore the sets $M_{w} \subset L_{w}$ consisting 
of decompositions that satisfy $\hat{s}\left(x_{i}, y_{i}\right)=w-1$ are in fact line segments parallel to the kernel of $\hat{s}$. The generalization axiom requires that for any $\left(x_{i}, y_{i}\right)=\left(x_{i}, w x_{i}^{-1}\right) \in M_{w}$ we have $\sum_{i=1}^{n} \alpha_{i}^{0}\left(x_{i}, y_{i}\right)=\sum_{i=1}^{n} \alpha_{i}^{0}\left(x_{i}, w x_{i}^{-1}\right)=\left(\prod_{i=1}^{n} x_{i}^{\alpha_{i}}, w \prod_{i=1}^{n} x_{i}^{-\alpha_{i}}\right)$. But this means that for any fixed $w$ the function mapping each $\left(x, w x^{-1}\right) \in M_{w}$ to $x \in \Omega$ must be an affine map and in fact each $M_{w}=K_{w}$. Therefore we may state that a quasilinear index number pair is a generalization of the geometric mean if and only if each of the lines $K_{w}$ are affine copies of the multiplicative space $\Omega$. In the next section we will discuss the geometric interpretation of this.

Let $d$ be an additive decomposition function which represents a normalized generalization of the geometric mean. Then for any fixed $w \in \Omega$ the map $g_{w}\left(x, w x^{-1}\right) \longmapsto x$ is an affine bijection $\hat{g}_{w}$ from the line $F_{w}$ defined by the equation $\hat{s}(z)=w-1$ to the multiplicative space $\Omega$. As $d$ is a restriction of a linear map $D \rightarrow \mathbb{R}^{2}$ and the exponential function is a linear map $\mathbb{R} \rightarrow$ the composite $\hat{m}_{w}=\hat{d} \circ \hat{g}_{w} \circ \exp$ maps $\mathbb{R}$ affinely to a line in $\mathbb{R}^{2}$ for each fixed $w$. Noting that $\log x=\log g_{w}\left(x, w x^{-1}\right)$ and thus $d\left(x, w x^{-1}\right)=\hat{m}_{w}(\log x)$ for any $w$, we see that $d$ must be of the form $d\left(x, w x^{-1}\right)=\left[\begin{array}{l}a_{1}(w) \\ a_{2}(w)\end{array}\right]+\left[\begin{array}{l}b_{1}(w) \\ b_{2}(w)\end{array}\right] \log x$ for all $w$ and $x$. Substituting $w=x y$ we have $d_{1}(x, y)=a_{1}(x y)+b_{1}(x y) \log x$. Normalization yields $a_{1}(x)=d_{1}(1, x)=0$ and $a_{1}(x)+b_{1}(x) \log x=x-1$ which imply that $d_{1}(x, y)=\frac{x y-1}{\log x y} \log x=l(x y) \log x$. As $d$ is an additive decomposition function, $d_{2}(x, y)=x y-1-d_{1}(x, y)=l(x y) \log y$. This can be achieved by choosing $a_{2}(z)=z-1$ and $b_{2}(z)=-b_{1}(z)$ so that $d$ is of the required form. Thus $d$ is the additive representation of the Montgomery-Vartia index number pair as given in Table 1 . 